\title{
Linked partition ideals, directed graphs and $q$-multi-summations
}

\author{
Shane Chern \\ Department of Mathematics \\ Penn State University \\ University Park, PA, U.S.A. \\ shanechern@psu.edu
}

Submitted: Mar 17, 2020; Accepted: Aug 8, 2020; Published: Aug 21, 2020

(C) The authors. Released under the CC BY-ND license (International 4.0).

\begin{abstract}
In this paper, we start by considering generating function identities for linked partition ideals in the setting of basic graph theory. Then our attention is turned to $q$-difference systems, which eventually lead to a factorization problem of a special type of column functional vectors involving $q$-multi-summations. Using a recurrence relation satisfied by certain $q$-multi-summations, we are able to provide non-computer-assisted proofs of some Andrews-Gordon type generating function identities. These proofs also have an interesting connection with binary trees. Further, we give illustrations of constructing a linked partition ideal, or more loosely, a set of integer partitions whose generating function corresponds to a given set of special $q$-multi-summations.
\end{abstract}

Mathematics Subject Classifications: 11P84, 05A17, 05C05, 05C20, 33D70

\section{Introduction}

\subsection{Rogers-Ramanujan type identities}

The two Rogers-Ramanujan identities [22, 24], which read as follows, have attracted a great deal of research interest in the theory of partitions.

Theorem 1 (Rogers-Ramanujan identities).

(i) The number of partitions of a nonnegative integer $n$ into parts congruent to \pm 1 modulo 5 is the same as the number of partitions of $n$ such that the adjacent parts differ by at least 2 . 
(ii) The number of partitions of a nonnegative integer $n$ into parts congruent to \pm 2 modulo 5 is the same as the number of partitions of $n$ such that the adjacent parts differ by at least 2 and such that the smallest part is at least 2.

There are many identities of the same flavor, including the Andrews-Gordon identity [2, 12], the Göllnitz-Gordon identities [11, 13], the Capparelli identities [8] and so forth. In 2014, Kanade and Russell [16] further proposed six challenging conjectures on RogersRamanujan type identities, the latter two of which were proved in 2018 by Bringmann, Jennings-Shaffer and Mahlburg [7].

Among these Rogers-Ramanujan type identities, two types of partition sets are considered. One partition set consists of partitions under certain congruence condition. For example, in the first Rogers-Ramanujan identity, we enumerate partitions into parts congruent to \pm 1 modulo 5. The other partition set contains partitions under certain difference-at-a-distance theme. Let us first adopt a definition in [16].

Definition 2. We say that a partition $\lambda=\lambda_{1}+\lambda_{2}+\cdots+\lambda_{\ell}$ satisfies the difference at least $d$ at distance $k$ condition if, for all $j, \lambda_{j}-\lambda_{j+k} \geqslant d$.

In this setting, we may paraphrase the corresponding partition set in the first RogersRamanujan identity as the set of partitions with difference at least 2 at distance 1.

Although it is straightforward to find the generating function for partitions under a given congruence condition, it is generally not easy to obtain an analytic form of generating function for partitions under a difference-at-a-distance theme - this is why the six conjectures of Kanade and Russell remained mysterious for years. But this problem was recently settled by Kanade and Russell themselves [17] and independently by Kurşungöz [19] using combinatorial approaches. Also, Kurşungöz carried out similar treatments for Capparelli's and Göllnitz-Gordon identities in [20]. Later Li and the author [9] proposed an algebraic method to reprove six Andrews-Gordon type generating function identities related to the Kanade-Russell conjectures and also to discover a number of new identities.

For example, in the Kanade-Russell conjecture $I_{1}$, we would like to count

"partitions with difference at least 3 at distance 2 such that if two consecutive parts differ by at most 1 , then their sum is divisible by $3 . "$

It was shown in [19] that its generating function is a double summation as follows:

$$
\sum_{\lambda} x^{\sharp(\lambda)} q^{|\lambda|}=\sum_{n_{1}, n_{2} \geqslant 0} \frac{q^{n_{1}^{2}+3 n_{2}^{2}+3 n_{1} n_{2}} x^{n_{1}+2 n_{2}}}{(q ; q)_{n_{1}}\left(q^{3} ; q^{3}\right)_{n_{2}}},
$$

where $\lambda$ runs through all such partitions, $\sharp(\lambda)$ denotes the number of parts in $\lambda$ and $|\lambda|$ is the size of $\lambda$ (that is, the sum of all parts in $\lambda$ ).

\subsection{Span one linked partition ideals}

In the 1970s, George Andrews [3, 4, 5] has already started a systematic study of RogersRamanujan type identities and developed a general theory in which the concept of linked 
partition ideals was introduced. The interested readers may refer to Chapter 8 of Andrews' monograph: The theory of partitions [6].

What we are interested in this paper is a special case of linked partition ideals - the span one linked partition ideals. In fact, this special case is enough to cover most partition sets under difference-at-a-distance themes.

Let us first fix some notations.

Let $\mathscr{P}$ be the set of all integer partitions with the empty partition $\varnothing$ included. We adopt the convention that $|\varnothing|=0$ and $\sharp(\varnothing)=0$. We define a map $\phi: \mathscr{P} \rightarrow \mathscr{P}$ by sending a partition $\lambda$ to another partition which is obtained by adding 1 to each part of $\lambda$. Also, we stipulate that $\phi(\varnothing)=\varnothing$. For example, $\phi(5+3+3+2+1)=6+4+4+3+2$. For $k \geqslant 1$, we iteratively write $\phi^{k}(\lambda)=\phi\left(\phi^{k-1}(\lambda)\right)$ with $\phi^{0}(\lambda)=\lambda$. Also, for two partitions $\lambda$ and $\pi$, their sum $\lambda \oplus \pi$ is constructed by collecting all parts in $\lambda$ and $\pi$ in weakly decreasing order. For example, if $\lambda=3+2+1+1$ and $\pi=4+2+2+1+1$, then $\lambda \oplus \pi=4+3+2+2+2+1+1+1+1$.

Let $\Pi$ be a finite set of partitions containing the empty partition $\varnothing$. For each partition $\pi \in \Pi$, we define its linking set $\mathcal{L}(\pi)$ by a subset of $\Pi$ containing the empty partition $\varnothing$. Also, we require that the linking set of the empty partition, $\mathcal{L}(\varnothing)$, equals $\Pi$. It is possible to construct finite chains

$$
\lambda_{0} \rightarrow \lambda_{1} \rightarrow \lambda_{2} \rightarrow \cdots \rightarrow \lambda_{K}
$$

such that $\lambda_{0} \in \Pi, \lambda_{K} \neq \varnothing$ and for all $1 \leqslant k \leqslant K, \lambda_{k} \in \mathcal{L}\left(\lambda_{k-1}\right)$. We may further extend such a finite chain to an infinite chain ending with a series of empty partitions

$$
\mathcal{C}: \lambda_{0} \rightarrow \lambda_{1} \rightarrow \lambda_{2} \rightarrow \cdots \rightarrow \lambda_{K} \rightarrow \varnothing \rightarrow \varnothing \rightarrow \cdots
$$

Let $S$ be a positive integer no smaller than the largest part among all partitions in $\Pi$. The above infinite chain $\mathcal{C}$ uniquely determines a partition by

$$
\lambda_{0} \oplus \phi^{S}\left(\lambda_{1}\right) \oplus \phi^{2 S}\left(\lambda_{2}\right) \oplus \cdots \oplus \phi^{K S}\left(\lambda_{K}\right) \oplus \phi^{(K+1) S}(\varnothing) \oplus \phi^{(K+2) S}(\varnothing) \oplus \cdots,
$$

which is equivalent to

$$
\lambda_{0} \oplus \phi^{S}\left(\lambda_{1}\right) \oplus \phi^{2 S}\left(\lambda_{2}\right) \oplus \cdots \oplus \phi^{K S}\left(\lambda_{K}\right) .
$$

Let us collect such partitions along with the empty partition $\lambda=\varnothing$ (which corresponds to the infinite chain $\varnothing \rightarrow \varnothing \rightarrow \cdots)$ and obtain a partition set $\mathscr{I}:=\mathscr{I}(\langle\Pi, \mathcal{L}\rangle, S)$. Then $\mathscr{I}$ is called a span one linked partition ideal.

Example 3. In the first Rogers-Ramanujan identity, we consider partitions with difference at least 2 at distance 1 . It is not hard to verify that this partition set is a span one linked partition ideal $\mathscr{I}(\langle\Pi, \mathcal{L}\rangle, S)$ where $\Pi=\{\varnothing, 1,2\}$ in which 1 denotes an integer partition containing one part of size 1 and likewise 2 denotes an integer partition containing one part of size 2 , the linking sets are

$$
\mathcal{L}(\varnothing)=\{\varnothing, 1,2\}, \quad \mathcal{L}(1)=\{\varnothing, 1,2\}, \quad \mathcal{L}(2)=\{\varnothing, 2\}
$$

and $S=2$. 


\subsection{Generating function for span one linked partition ideals}

Given a span one linked partition ideal $\mathscr{I}=\mathscr{I}(\langle\Pi, \mathcal{L}\rangle, S)$, one crucial problem is to determine its generating function

$$
\mathscr{G}(x)=\mathscr{G}(x, q):=\sum_{\lambda \in \mathscr{I}} x^{\sharp(\lambda)} q^{|\lambda|} .
$$

Assume that $\Pi=\left\{\pi_{1}, \pi_{2}, \ldots, \pi_{K}\right\}$ where $\pi_{1}=\varnothing$, the empty partition. We define a $(0,1)$-matrix $\mathscr{A}=\mathscr{A}(\langle\Pi, \mathcal{L}\rangle)$ by

$$
\mathscr{A}_{i, j}= \begin{cases}1 & \text { if } \pi_{j} \in \mathcal{L}\left(\pi_{i}\right), \\ 0 & \text { if } \pi_{j} \notin \mathcal{L}\left(\pi_{i}\right),\end{cases}
$$

and a diagonal matrix $\mathscr{W}(x)=\mathscr{W}(\langle\Pi, \mathcal{L}\rangle \mid x, q)$ by

$$
\mathscr{W}(x)=\left(\begin{array}{cccc}
x^{\sharp\left(\pi_{1}\right)} q^{\left|\pi_{1}\right|} & & & \\
& x^{\sharp\left(\pi_{2}\right)} q^{\left|\pi_{2}\right|} & & \\
& & \ddots & \\
& & & x^{\sharp\left(\pi_{K}\right)} q^{\left|\pi_{K}\right|}
\end{array}\right) .
$$

Let the $S$-tail of a partition $\lambda$ be the collection of parts in $\lambda$ that are at most $S$.

Theorem 4. For each $1 \leqslant k \leqslant K$, we denote by $\mathscr{I}_{k}$ the subset of partitions $\lambda$ in $\mathscr{I}(\langle\Pi, \mathcal{L}\rangle, S)$ whose $S$-tail is $\pi_{k} \in \Pi$. We further write

$$
\mathscr{G}_{k}(x)=\mathscr{G}_{k}(x, q):=\sum_{\lambda \in \mathscr{I}_{k}} x^{\sharp(\lambda)} q^{|\lambda|} .
$$

Let $\mathscr{A}$ and $\mathscr{W}(x)$ be defined as in (6) and (7), respectively. Then, for $|q|<1$ and $|x|<|q|^{-1}$,

$$
\left(\begin{array}{c}
\mathscr{G}_{1}(x) \\
\mathscr{G}_{2}(x) \\
\vdots \\
\mathscr{G}_{K}(x)
\end{array}\right)=\mathscr{W}(x) \cdot\left(\lim _{M \rightarrow \infty} \prod_{m=1}^{M}\left(\mathscr{A} \cdot \mathscr{W}\left(x q^{m S}\right)\right)\right) \cdot\left(\begin{array}{c}
1 \\
0 \\
\vdots \\
0
\end{array}\right) .
$$

Remark 5. Recall that $\pi_{1}=\varnothing\left(\right.$ so that $\pi_{1} \in \mathcal{L}(\pi)$ for all $\left.\pi \in \Pi\right)$ and $\mathcal{L}(\varnothing)=\Pi$. It follows that all entries in the first row and column of $\mathscr{A}$ are 1 . Further, the first entry in $\mathscr{W}(x)$ is also $x^{0} q^{0}=1$. When $|q|<1$ and $|x|<|q|^{-1}$, we have

$$
\lim _{M \rightarrow \infty} \mathscr{A} \cdot \mathscr{W}\left(x q^{M S}\right)=\left(\begin{array}{ccccc}
1 & 0 & 0 & \cdots & 0 \\
1 & 0 & 0 & \cdots & 0 \\
\vdots & \vdots & \vdots & \ddots & \vdots \\
1 & 0 & 0 & \cdots & 0
\end{array}\right)
$$

Throughout, $\prod_{m=1}^{M}\left(\mathscr{A} \cdot \mathscr{W}\left(x q^{m S}\right)\right)$ means

$$
\mathscr{A} . \mathscr{W}\left(x q^{S}\right) \cdot \mathscr{A} \cdot \mathscr{W}\left(x q^{2 S}\right) . \cdots \mathscr{A} \cdot \mathscr{W}\left(x q^{M S}\right) .
$$


Remark 6. We have

$$
\mathscr{G}(x)=\sum_{k=1}^{K} \mathscr{G}_{k}(x),
$$

but since $\mathcal{L}(\varnothing)=\Pi$, it is not hard to see that

$$
\mathscr{G}_{1}(x)=\sum_{k=1}^{K} \mathscr{G}_{k}\left(x q^{S}\right)
$$

Hence,

$$
\mathscr{G}(x)=\mathscr{G}_{1}\left(x q^{-S}\right) .
$$

In September 2018, George Andrews communicated to Zhitai Li and the author a conjecture on the generating function for linked partition ideals, which was recorded in $[9]$.

Conjecture 7 (Andrews). Every linked partition ideal has a bivariate generating function of the form

$$
\sum_{n_{1}, \ldots, n_{r} \geqslant 0} \frac{(-1)^{L_{1}\left(n_{1}, \ldots, n_{r}\right)} q^{Q\left(n_{1}, \ldots, n_{r}\right)+L_{2}\left(n_{1}, \ldots, n_{r}\right)} x^{L_{3}\left(n_{1}, \ldots, n_{r}\right)}}{\left(q^{B_{1}} ; q^{A_{1}}\right)_{n_{1}} \cdots\left(q^{B_{r}} ; q^{A_{r}}\right)_{n_{r}}}
$$

in which $L_{1}, L_{2}$ and $L_{3}$ are linear forms in $n_{1}, \ldots, n_{r}$ and $Q$ is a quadratic form in $n_{1}, \ldots, n_{r}$. Here the coefficient of the $x^{m} q^{n}$ term is the number of partitions of $n$ in this linked partition ideal with exactly $m$ parts.

By examining a number of examples in $[9,17,19,20]$, it seems that in some cases the $\mathscr{G}_{k}(x)$ 's in Theorem 4 are of a unified form of $q$-multi-summations. It motivates us to consider a matrix factorization problem involving column functional vectors of certain $q$ multi-summations. This, in turn, provides some crude ideas for the conjecture of Andrews.

Further, the algebraic method in [9] of proving generating function identities such as (1) relies heavily on computer algebra (Mathematica packages qMultiSum [23] and qGeneratingFunctions [18]). Now we are able to present a new approach that may simplify the considerations related to computer assistance.

It should be admitted that when a span one linked partition ideal is given, to derive an Andrews-Gordon type generating function identity, one still has to obtain first a conjectural $(x, q)$ sum-side. This then requires an extensive search using the general shape given by Andrews' Conjecture 7; see Section 5 in the work of Li and the author [9] for some discussions.

However, we could also start in the opposite direction. That is, if we are given a family of nice $q$-multi-summations, then we may try to use the approach in Section 4 to construct identities like (50) and (58), from which we may further construct some combinatorial objects, or even more luckily, a span one linked partition ideal and its subsets, such that the $q$-multi-summations correspond to their generating functions. One such instance is given in Theorem 23 and Corollary 25. This is indeed what we hope the framework in this paper could provide. 


\subsection{Outline of this paper}

This paper is organized as follows.

In Section 2, we first define the generating function for walks in a directed graph $G$. Then, by assigning an empty vertex to $G$, we obtain a modified directed graph $G^{!}$. The generating function attached to $G^{!}$can be defined naturally. Now we merely need to define the associated directed graph of a span one linked partition ideal $\mathscr{I}(\langle\Pi, \mathcal{L}\rangle, S)$ and then deduce Theorem 4 from the generating function attached to this associated directed graph.

In Section 3, we will study a $q$-difference system arising from Theorem 4. Two examples will then be discussed: one example comes from the Rogers-Ramanujan identities and the other is about the Kanade-Russell conjectures $I_{1}-I_{3}$. Then, a matrix factorization problem will be identified from the two examples.

In Section 4, we turn to non-computer-assisted proofs of two identities obtained in Section 3. The two identities, in turn, can be used to prove Andrews-Gordon type generating function identities for span one linked partition ideals. Our approach relies on a key recurrence relation obtained in Section 4.1. Also, we are able to elucidate the proofs by binary trees. Further, as an illustration of the framework in this paper, we will construct a set of integer partitions whose generating function corresponds to a set of $q$-multi-summations in Theorem 23 and Corollary 25.

Finally, we are going to raise some open problems in Section 5.

\section{Directed graphs}

Let $G=(V, E)$ be a directed graph where $V$ is the set of vertices and $E$ is the set of directed edges. Throughout, we allow loops (that is, directed edges connecting vertices with themselves) in $G$ but for any two vertices $u$ and $v$, not necessarily distinct, we allow at most one directed edge connecting $u$ with $v$. Let $V=\left\{v_{1}, v_{2}, \ldots, v_{K}\right\}$. Let $\mathscr{A}=\mathscr{A}(G)$ be the adjacency matrix of $G$, that is,

$$
\mathscr{A}_{i, j}= \begin{cases}1 & \text { if there is a directed edge from } v_{i} \text { with } v_{j}, \\ 0 & \text { if there are no directed edges from } v_{i} \text { with } v_{j}\end{cases}
$$

We say that $w$ is a walk of step $M$ in $G$ if $w$ is a chain of $M+1$ vertices

$$
\varpi_{0} \rightarrow \varpi_{1} \rightarrow \cdots \rightarrow \varpi_{M}
$$

such that for each $1 \leqslant m \leqslant M$, there is an edge from $\varpi_{m-1}$ to $\varpi_{m}$. Let $\mathcal{W}_{M}$ be the set of walks of step $M$ in $G$.

\subsection{Generating function for walks in a directed graph}

To define the generating function for step $M$ walks in a directed graph $G=(V, E)$, we assign two weights to each vertex $v$ : one is called length, denoted by $\sharp(v) \in \mathbb{N}$, and the other is called size, denoted by $|v| \in \mathbb{N}$. 
Let the shift $S$ be a nonnegative integer.

For any walk $w \in \mathcal{W}_{M}$,

$$
w=\varpi_{0} \rightarrow \varpi_{1} \rightarrow \cdots \rightarrow \varpi_{M}
$$

we define its generating function by

$$
\mathscr{G}(w \mid x, q):=x^{\sharp\left(\varpi_{0}\right)} q^{\left|\varpi_{0}\right|} \times\left(x q^{S}\right)^{\sharp\left(\varpi_{1}\right)} q^{\left|\varpi_{1}\right|} \times \cdots \times\left(x q^{M S}\right)^{\sharp\left(\varpi_{M}\right)} q^{\left|\varpi_{M}\right|} .
$$

Now we are able to define the generating function for step $M$ walks from $v_{i}$ to $v_{j}$ for any $1 \leqslant i, j \leqslant K$ :

$$
\mathscr{G}_{i, j}\left(\mathcal{W}_{M} \mid x\right)=\mathscr{G}_{i, j}\left(\mathcal{W}_{M} \mid x, q\right):=\sum_{\substack{w \in \mathcal{W}_{M} \\ \varpi_{0}=v_{i} \\ \varpi_{M}=v_{j}}} \mathscr{G}(w \mid x, q)
$$

Let us define a diagonal matrix $\mathscr{W}(x)=\mathscr{W}(x, q)$ by

$$
\mathscr{W}(x)=\left(\begin{array}{llll}
x^{\sharp\left(v_{1}\right)} q^{\left|v_{1}\right|} & & & \\
& x^{\sharp\left(v_{2}\right)} q^{\left|v_{2}\right|} & & \\
& & \ddots & \\
& & & x^{\sharp\left(v_{K}\right)} q^{\left|v_{K}\right|}
\end{array}\right) .
$$

Theorem 8. Let $\mathscr{A}$ be the adjacency matrix of $G$ and let $\mathscr{W}(x)$ be as in (16). Then $\mathscr{G}_{i, j}\left(\mathcal{W}_{M} \mid x\right)$ is the $(i, j)$-th entry of

$$
\mathscr{W}(x) \cdot \mathscr{A} \cdot \mathscr{W}\left(x q^{S}\right) \cdot \mathscr{A} \cdot \mathscr{W}\left(x q^{2 S}\right) . \cdots \cdot \mathscr{A} \cdot \mathscr{W}\left(x q^{M S}\right) .
$$

Remark 9. Let us set $x=q=1$. Then $\mathscr{W}(1,1)$ is a $K \times K$ identity matrix and hence (17) becomes $\mathscr{A}^{M}$. Since $\mathscr{G}_{i, j}\left(\mathcal{W}_{M} \mid 1,1\right)$ equals the number of walks of step $M$ from vertex $v_{i}$ to vertex $v_{j}$, Theorem 8 immediately leads to a well-known result in graph theory:

Corollary 10. The number of walks of step $M$ from vertex $v_{i}$ to vertex $v_{j}$ is the $(i, j)$-th entry of $\mathscr{A}^{M}$.

Proof of Theorem 8. We induct on $M$. When $M=0$, that is, the chain $w$ of vertices in (13) contains only one vertex $\varpi_{0}$, it follows that

$$
\mathscr{G}_{i, j}\left(\mathcal{W}_{0} \mid x\right)= \begin{cases}x^{\sharp\left(v_{i}\right)} q^{\left|v_{i}\right|} & \text { if } i=j, \\ 0 & \text { if } i \neq j,\end{cases}
$$

which is identical to the $(i, j)$-th entry of $\mathscr{W}(x)$.

Now let us assume that the theorem is true for some $M \geqslant 0$. We also write for convenience

$$
\mathscr{M}(M)=\mathscr{W}(x) \cdot \mathscr{A} \cdot \mathscr{W}\left(x q^{S}\right) \cdot \mathscr{A} \cdot \mathscr{W}\left(x q^{2 S}\right) . \cdots \cdot \mathscr{A} \cdot \mathscr{W}\left(x q^{M S}\right)
$$


Then $\mathscr{G}_{i, j}\left(\mathcal{W}_{M} \mid x\right)=\mathscr{M}(M)_{i, j}$. Further,

$$
\begin{aligned}
\mathscr{M}(M+1)_{i, j} & =\sum_{k=1}^{K} \mathscr{M}(M)_{i, k} \mathscr{A}_{k, j}\left(x q^{(M+1) S}\right)^{\sharp\left(v_{j}\right)} q^{\left|v_{j}\right|} \\
& =\sum_{k=1}^{K} \mathscr{G}_{i, k}\left(\mathcal{W}_{M} \mid x\right) \mathscr{A}_{k, j}\left(x q^{(M+1) S}\right)^{\sharp\left(v_{j}\right)} q^{\left|v_{j}\right|} .
\end{aligned}
$$

On the other hand,

$$
\begin{aligned}
\mathscr{G}_{i, j}\left(\mathcal{W}_{M+1} \mid x\right) & =\sum_{\substack{w \in \mathcal{W}_{M+1} \\
\varpi_{0}=v_{i} \\
\varpi_{M}=v_{j}}} \mathscr{G}(w \mid x, q) \\
& =\sum_{k=1}^{K}\left(\sum_{\begin{array}{c}
w \in \mathcal{W}_{M} \\
\varpi_{0}=v_{i} \\
\varpi_{M}=v_{k}
\end{array}} \mathscr{G}(w \mid x, q)\right) \mathscr{A}_{k, j}\left(x q^{(M+1) S}\right)^{\sharp\left(v_{j}\right)} q^{\left|v_{j}\right|} \\
& =\sum_{k=1}^{K} \mathscr{G}_{i, k}\left(\mathcal{W}_{M} \mid x\right) \mathscr{A}_{k, j}\left(x q^{(M+1) S}\right)^{\sharp\left(v_{j}\right)} q^{\left|v_{j}\right|} .
\end{aligned}
$$

Hence, $\mathscr{G}_{i, j}\left(\mathcal{W}_{M+1} \mid x\right)=\mathscr{M}(M+1)_{i, j}$, which is our desired result.

\subsection{Assigning an empty vertex}

Let us assume that $v_{1} \in V$ is an empty vertex, that is, its length and size are both 0 :

$$
\sharp\left(v_{1}\right)=0 \text { and }\left|v_{1}\right|=0 .
$$

We also assume that, for $2 \leqslant k \leqslant K, \sharp\left(v_{k}\right)$ and $\left|v_{k}\right|$ are both positive integers.

Now we stipulate that, for each $1 \leqslant k \leqslant K$, there is an edge from vertex $v_{k}$ to the empty vertex $v_{1}$. Hence, the entries in the first column of the adjacency matrix $\mathscr{A}$ are all 1 .

We call such modified directed graph $G^{!}=\left(V^{!}, E^{!}\right)$.

For any finite walk in $G^{!}$,

$$
w=\varpi_{0} \rightarrow \varpi_{1} \rightarrow \cdots \rightarrow \varpi_{M}
$$

with $\varpi_{M} \neq v_{1}$, we may extend it to an infinite walk

$$
w^{\star}=\varpi_{0} \rightarrow \varpi_{1} \rightarrow \cdots \rightarrow \varpi_{M} \rightarrow v_{1} \rightarrow v_{1} \rightarrow \cdots .
$$

Conversely, for any infinite walk $w^{\star}$ in $G^{!}$ending with $v_{1} \rightarrow v_{1} \rightarrow \cdots$, a series of empty vertex, we may find the last vertex, say $\varpi_{M}$, which is not empty, and reduce $w^{\star}$ to a finite walk $w=\varpi_{0} \rightarrow \varpi_{1} \rightarrow \cdots \rightarrow \varpi_{M}$. If there is no such $\varpi_{M}$, that is, if the infinite walk is $v_{1} \rightarrow v_{1} \rightarrow \cdots$, we reduce it to $v_{1}$. 
It follows from the assumptions $\sharp\left(v_{1}\right)=0$ and $\left|v_{1}\right|=0$ that

$$
\mathscr{G}\left(w^{\star} \mid x, q\right)=\mathscr{G}(w \mid x, q) .
$$

Also, for the infinite walk $v_{1} \rightarrow v_{1} \rightarrow \cdots$, we have

$$
\mathscr{G}\left(v_{1} \rightarrow v_{1} \rightarrow \cdots \mid x, q\right)=\mathscr{G}\left(v_{1} \mid x, q\right)=x^{0} q^{0}=1 .
$$

Let $\mathcal{W}^{\star}$ denote the set of infinite walks in $G^{!}$ending with $v_{1} \rightarrow v_{1} \rightarrow \cdots$, a series of empty vertex.

We are now in the position to define the generating function attached to $G^{!}$, by

$$
\mathscr{G}\left(G^{!} \mid x, q\right):=\sum_{w^{\star} \in \mathcal{W}^{\star}} \mathscr{G}\left(w^{\star} \mid x, q\right) .
$$

Theorem 11. For each $1 \leqslant k \leqslant K$, let $\mathscr{G}_{k}\left(G^{!} \mid x\right)=\mathscr{G}_{k}\left(G^{!} \mid x, q\right)$ denote the generating function for infinite walks in $\mathcal{W}^{\star}$ starting at $v_{k}$. Let the shift $S$ be a positive integer. Let $\mathscr{A}$ and $\mathscr{W}(x)$ be defined as in (12) and (16), respectively. Then, for $|q|<1$ and $|x|<|q|^{-1}$,

$$
\left(\begin{array}{c}
\mathscr{G}_{1}\left(G^{!} \mid x\right) \\
\mathscr{G}_{2}\left(G^{!} \mid x\right) \\
\vdots \\
\mathscr{G}_{K}\left(G^{!} \mid x\right)
\end{array}\right)=\mathscr{W}(x) \cdot\left(\lim _{M \rightarrow \infty} \prod_{m=1}^{M}\left(\mathscr{A} \cdot \mathscr{W}\left(x q^{m S}\right)\right)\right) \cdot\left(\begin{array}{c}
1 \\
0 \\
\vdots \\
0
\end{array}\right)
$$

Proof. We simply observe that, for each $1 \leqslant k \leqslant K$,

$$
\mathscr{G}_{k}\left(G^{!} \mid x\right)=\lim _{M \rightarrow \infty} \sum_{\substack{w \in \mathcal{W}_{M} \\ \varpi_{0}=v_{k} \\ \varpi_{M}=v_{1}}} \mathscr{G}(w \mid x, q) .
$$

By Theorem 8 , this is the $(k, 1)$-th entry of

$$
\mathscr{W}(x) . \mathscr{A} \cdot \mathscr{W}\left(x q^{S}\right) \cdot \mathscr{A} \cdot \mathscr{W}\left(x q^{2 S}\right) . \cdots=\mathscr{W}(x) .\left(\lim _{M \rightarrow \infty} \prod_{m=1}^{M}\left(\mathscr{A} \cdot \mathscr{W}\left(x q^{m S}\right)\right)\right)
$$

The desired result therefore follows.

Remark 12. Results of the same flavor as Theorem 11 are available in literature for some other concrete identities; see [14, Section 3] for Gordon's identities, [10, Section 5] for the Andrews-Göllnitz-Gordon identities, and [15, Section 6] for the Andrews-Bressoud identities. 


\subsection{Proof of Theorem 4}

To prove Theorem 4, let us define the associated directed graph of a span one linked partition ideal $\mathscr{I}=\mathscr{I}(\langle\Pi, \mathcal{L}\rangle, S)$.

We first define the set of vertices. Since $\Pi=\left\{\pi_{1}, \pi_{2}, \ldots, \pi_{K}\right\}$ is a finite set of partitions, we may treat each $\pi_{k}$ as a vertex. We also define the length of $\pi_{k}$ as the number of parts in $\pi_{k}$ and the size of $\pi_{k}$ as the sum of all parts in $\pi_{k}$. In particular, since $\pi_{1}$ is an empty partition so that $\sharp\left(\pi_{1}\right)=0$ and $\left|\pi_{1}\right|=0$, we may treat $\pi_{1}$ as an empty vertex.

We next define the directed edges in a natural way. For $1 \leqslant i, j \leqslant K$, if $\pi_{j} \in \mathcal{L}\left(\pi_{i}\right)$, then we say that there is an edge from vertex $\pi_{i}$ to vertex $\pi_{j}$. Recall that for any $\pi \in \Pi$, its linking set $\mathcal{L}(\pi)$ is defined to contain the empty partition $\pi_{1}=\varnothing$. Hence, for each $1 \leqslant k \leqslant K$, there is an edge from vertex $\pi_{k}$ to vertex $\pi_{1}$.

We call this graph the associated directed graph of $\mathscr{I}$, denoted by $G^{!}(\mathscr{I})=\left(V^{!}(\mathscr{I})\right.$, $\left.E^{!}(\mathscr{I})\right)$. In fact, $G^{!}(\mathscr{I})$ is a modified directed graph described in Section 2.2.

Recall from (4) that each partition $\lambda$ in $\mathscr{I}$ can be uniquely decomposed as

$$
\lambda=\lambda_{0} \oplus \phi^{S}\left(\lambda_{1}\right) \oplus \phi^{2 S}\left(\lambda_{2}\right) \oplus \cdots \oplus \phi^{K S}\left(\lambda_{K}\right) \oplus \phi^{(K+1) S}(\varnothing) \oplus \phi^{(K+2) S}(\varnothing) \oplus \cdots
$$

so that $\lambda_{K} \neq \varnothing$ as long as $\lambda \neq \varnothing$. Hence, we have a natural bijection to infinite walks in $G^{!}(\mathscr{I})$ ending with $\pi_{1} \rightarrow \pi_{1} \rightarrow \cdots$ :

$$
w^{\star}(\lambda)=\lambda_{0} \rightarrow \lambda_{1} \rightarrow \lambda_{2} \rightarrow \cdots \rightarrow \lambda_{K} \rightarrow \pi_{1} \rightarrow \pi_{1} \rightarrow \cdots
$$

Further, if $\lambda$ is an empty partition, then the resulted infinite walk is simply $\pi_{1} \rightarrow \pi_{1} \rightarrow \cdots$.

Now let us define $S$ to be the shift. Then

$$
x^{\sharp(\lambda)} q^{|\lambda|}=\mathscr{G}\left(w^{\star}(\lambda) \mid x, q\right) .
$$

Hence,

$$
\mathscr{G}(x)=\sum_{\lambda \in \mathscr{I}} x^{\sharp(\lambda)} q^{|\lambda|}=\sum_{w^{\star} \in \mathcal{W}^{\star}} \mathscr{G}\left(w^{\star} \mid x, q\right) .
$$

The rest follows directly from Theorem 11 .

Example 13. It is shown in Example 3 that partitions with difference at least 2 at distance 1 form a span one linked partition ideal $\mathscr{I}(\langle\Pi, \mathcal{L}\rangle, S)$ where $\Pi=\{\varnothing, 1,2\}$, the linking sets are

$$
\mathcal{L}(\varnothing)=\{\varnothing, 1,2\}, \quad \mathcal{L}(1)=\{\varnothing, 1,2\}, \quad \mathcal{L}(2)=\{\varnothing, 2\}
$$

and $S=2$. We represent its associated directed graph in Fig. 1. 
Figure 1: The associated directed graph in Example 13

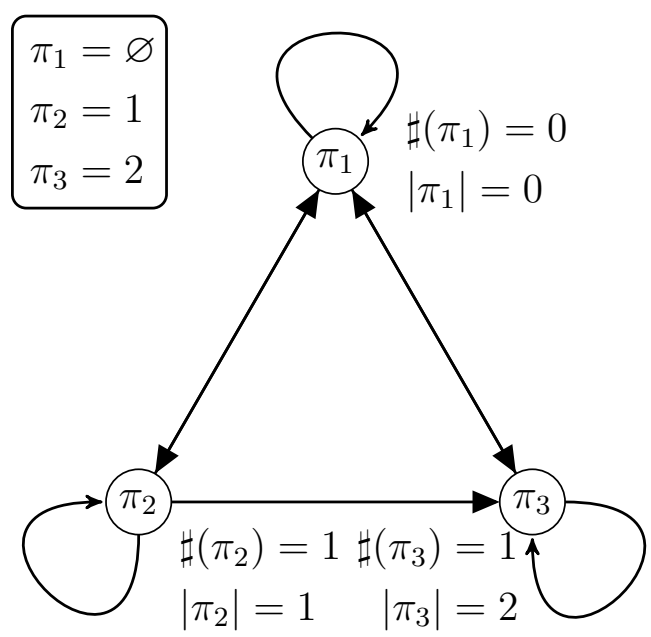

\section{$3 \quad q$-Multi-summations}

\subsection{A $q$-difference system and the uniqueness of solutions}

Recall that in Theorem 4 we have shown that

$$
\left(\begin{array}{c}
\mathscr{G}_{1}(x) \\
\mathscr{G}_{2}(x) \\
\vdots \\
\mathscr{G}_{K}(x)
\end{array}\right)=\mathscr{W}(x) \cdot\left(\lim _{M \rightarrow \infty} \prod_{m=1}^{M}\left(\mathscr{A} . \mathscr{W}\left(x q^{m S}\right)\right)\right) \cdot\left(\begin{array}{c}
1 \\
0 \\
\vdots \\
0
\end{array}\right) .
$$

Let us focus on

$$
\left(\begin{array}{c}
F_{1}^{\star}(x) \\
F_{2}^{\star}(x) \\
\vdots \\
F_{K}^{\star}(x)
\end{array}\right):=\left(\lim _{M \rightarrow \infty} \prod_{m=1}^{M}\left(\mathscr{A} \cdot \mathscr{W}\left(x q^{m S}\right)\right)\right) \cdot\left(\begin{array}{c}
1 \\
0 \\
\vdots \\
0
\end{array}\right)
$$

Notice that

$$
\begin{aligned}
\left(\begin{array}{c}
F_{1}^{\star}(x) \\
F_{2}^{\star}(x) \\
\vdots \\
F_{K}^{\star}(x)
\end{array}\right) & =\left(\lim _{M \rightarrow \infty} \prod_{m=1}^{M}\left(\mathscr{A} \cdot \mathscr{W}\left(x q^{m S}\right)\right)\right) \cdot\left(\begin{array}{c}
1 \\
0 \\
\vdots \\
0
\end{array}\right) \\
& =\mathscr{A} \cdot \mathscr{W}\left(x q^{S}\right) \cdot\left(\lim _{M \rightarrow \infty} \prod_{m=1}^{M}\left(\mathscr{A} \cdot \mathscr{W}\left(x q^{S} q^{m S}\right)\right)\right) \cdot\left(\begin{array}{c}
1 \\
0 \\
\vdots \\
0
\end{array}\right)
\end{aligned}
$$




$$
=\mathscr{A} \cdot \mathscr{W}\left(x q^{S}\right) \cdot\left(\begin{array}{c}
F_{1}^{\star}\left(x q^{S}\right) \\
F_{2}^{\star}\left(x q^{S}\right) \\
\vdots \\
F_{K}^{\star}\left(x q^{S}\right)
\end{array}\right)
$$

If we further write $F_{k}(x):=F_{k}^{\star}\left(x q^{-S}\right)$ for each $k$, then the column vector

$$
\underline{\mathbf{F}}(x):=\left(\begin{array}{c}
F_{1}(x) \\
F_{2}(x) \\
\vdots \\
F_{K}(x)
\end{array}\right)
$$

satisfies the $q$-difference system

$$
\underline{\mathbf{F}}(x)=\mathscr{A} \cdot \mathscr{W}(x) \cdot \underline{\mathbf{F}}\left(x q^{S}\right) .
$$

Remark 14. It follows from (23), (24) and (25) that

$$
\underline{\mathbf{F}}(x)=\mathscr{A} \cdot \mathscr{W}(x) .\left(\begin{array}{c}
F_{1}^{\star}(x) \\
F_{2}^{\star}(x) \\
\vdots \\
F_{K}^{\star}(x)
\end{array}\right)=\mathscr{A} \cdot\left(\begin{array}{c}
\mathscr{G}_{1}(x) \\
\mathscr{G}_{2}(x) \\
\vdots \\
\mathscr{G}_{K}(x)
\end{array}\right) .
$$

Recall that, we have defined in Theorem 4 that, for each $1 \leqslant k \leqslant K, \mathscr{I}_{k}$ denotes the subset of partitions in $\mathscr{I}(\langle\Pi, \mathcal{L}\rangle, S)$ whose $S$-tail is $\pi_{k}$. Further, $\mathscr{C}_{k}(x)$ is the generating function for $\mathscr{I}_{k}$. Since $\mathscr{A}$ is a $(0,1)$-matrix, it follows that $F_{k}(x) \in \mathbb{Z}[[q]][[x]]$ for each $1 \leqslant k \leqslant K$. More importantly, since the empty partition $\varnothing$ is contained in $\mathscr{I}_{1}$ but not in $\mathscr{I}_{k}$ for $2 \leqslant k \leqslant K$, we have $\mathscr{G}_{1}(0)=1$ and $\mathscr{G}_{k}(0)=0$ for $2 \leqslant k \leqslant K$. Since the entries in the first column of $\mathscr{A}$ are all 1 , it follows that

$$
F_{1}(0)=F_{2}(0)=\cdots=F_{K}(0)=1 .
$$

We next show the uniqueness of solutions of (25).

Proposition 15. In the q-difference system (25), we assume that, for each $1 \leqslant k \leqslant K$, $F_{k}(x) \in \mathbb{C}[[q]][[x]]$. If $F_{1}(0)=F_{2}(0)=\cdots=F_{K}(0)$, then there exists a solution to $(25)$. Further, the solution is uniquely determined by $\underline{\mathbf{F}}(0)$.

Proof. For each $1 \leqslant k \leqslant K$, let us write

$$
F_{k}(x)=\sum_{n \geqslant 0} f_{k}(n) x^{n}
$$

where $f_{k}(n) \in \mathbb{C}[[q]]$ for $n \geqslant 0$. We also write for notational convenience that $f_{k}(n)=0$ for $n<0$. Then,

$$
\sum_{n \geqslant 0} f_{k}(n) x^{n}=\sum_{j=1}^{K} \mathscr{A}_{k, j} x^{\sharp\left(\pi_{j}\right)} q^{\left|\pi_{j}\right|} \sum_{n \geqslant 0} f_{j}(n) q^{n S} x^{n}
$$




$$
=\sum_{n \geqslant 0}\left(\sum_{j=1}^{K} \mathscr{A}_{k, j} q^{\left|\pi_{j}\right|+\left(n-\sharp\left(\pi_{j}\right)\right) S} f_{j}\left(n-\sharp\left(\pi_{j}\right)\right)\right) x^{n} .
$$

Recall that $\sharp\left(\pi_{1}\right)=\left|\pi_{1}\right|=0$ and $\mathscr{A}_{k, 1}=1$ for all $k$. We have that, for $n \geqslant 0$,

$$
f_{k}(n)=q^{n S} f_{1}(n)+\sum_{j=2}^{K} \mathscr{A}_{k, j} q^{\left|\pi_{j}\right|+\left(n-\sharp\left(\pi_{j}\right)\right) S} f_{j}\left(n-\sharp\left(\pi_{j}\right)\right) .
$$

Setting $n=0$ gives the requirement $F_{1}(0)=F_{2}(0)=\cdots=F_{K}(0)$. Also, $\underline{\mathbf{F}}(0)=$ $\left(f_{1}(0), f_{2}(0), \ldots, f_{K}(0)\right)^{T}$ uniquely determines $f_{k}(n)$ for all $1 \leqslant k \leqslant K$ and $n \geqslant 1$ by $(28)$.

\subsection{Two examples}

Recall that, for each $1 \leqslant k \leqslant K, \mathscr{I}_{k}$ denotes the subset of partitions in $\mathscr{I}(\langle\Pi, \mathcal{L}\rangle, S)$ whose $S$-tail is $\pi_{k}$. Further,

$$
\mathscr{G}_{k}(x)=\sum_{\lambda \in \mathscr{I}_{k}} x^{\sharp(\lambda)} q^{|\lambda|} .
$$

\subsubsection{Example 1}

In the first example, we consider

"partitions with difference at least 2 at distance 1."

This partition set obviously corresponds to the Rogers-Ramanujan identities. In Example 3 , we have shown that it is a span one linked partition ideal $\mathscr{I}(\langle\Pi, \mathcal{L}\rangle, S)$ where $\Pi=$ $\left\{\pi_{1}, \pi_{2}, \pi_{3}\right\}$ with $\pi_{1}=\varnothing, \pi_{2}=1$ and $\pi_{3}=2$, the linking sets are

$$
\mathcal{L}\left(\pi_{1}\right)=\left\{\pi_{1}, \pi_{2}, \pi_{3}\right\}, \quad \mathcal{L}\left(\pi_{2}\right)=\left\{\pi_{1}, \pi_{2}, \pi_{3}\right\}, \quad \mathcal{L}\left(\pi_{3}\right)=\left\{\pi_{1}, \pi_{3}\right\},
$$

and $S=2$.

Notice that the generating function for partitions with difference at least 2 at distance 1 is

$$
\mathscr{G}_{1}(x)+\mathscr{G}_{2}(x)+\mathscr{G}_{3}(x)=\sum_{n \geqslant 0} \frac{q^{n^{2}} x^{n}}{(q ; q)_{n}}
$$

and that the generating function for partitions with difference at least 2 at distance 1 and the smallest part at least 2 is

$$
\mathscr{G}_{1}(x)+\mathscr{G}_{3}(x)=\sum_{n \geqslant 0} \frac{q^{n^{2}+n} x^{n}}{(q ; q)_{n}} .
$$

We know from (26) that

$$
\left(\begin{array}{l}
F_{1}(x) \\
F_{2}(x) \\
F_{3}(x)
\end{array}\right)=\mathscr{A} \cdot\left(\begin{array}{l}
\mathscr{G}_{1}(x) \\
\mathscr{G}_{2}(x) \\
\mathscr{G}_{3}(x)
\end{array}\right)=\left(\begin{array}{lll}
1 & 1 & 1 \\
1 & 1 & 1 \\
1 & 0 & 1
\end{array}\right) \cdot\left(\begin{array}{c}
\mathscr{G}_{1}(x) \\
\mathscr{G}_{2}(x) \\
\mathscr{G}_{3}(x)
\end{array}\right) .
$$


Hence, by (29) and (30), if we put

$$
F_{1}(x)=F_{2}(x)=\sum_{n \geqslant 0} \frac{q^{n^{2}} x^{n}}{(q ; q)_{n}}
$$

and

$$
F_{3}(x)=\sum_{n \geqslant 0} \frac{q^{n^{2}+n} x^{n}}{(q ; q)_{n}}
$$

then we have the following relation from (25):

$$
\left(\begin{array}{l}
F_{1}(x) \\
F_{2}(x) \\
F_{3}(x)
\end{array}\right)=\left(\begin{array}{lll}
1 & 1 & 1 \\
1 & 1 & 1 \\
1 & 0 & 1
\end{array}\right) \cdot\left(\begin{array}{lll}
1 & & \\
& x q & \\
& & x q^{2}
\end{array}\right) \cdot\left(\begin{array}{l}
F_{1}\left(x q^{2}\right) \\
F_{2}\left(x q^{2}\right) \\
F_{3}\left(x q^{2}\right)
\end{array}\right) .
$$

Conversely, if we are able to prove (33) directly (notice that $F_{1}(0)=F_{2}(0)=F_{3}(0)=$ 1), then by Remark 14 and Proposition 15, we can compute that

$$
\begin{aligned}
\left(\begin{array}{l}
\mathscr{G}_{1}(x) \\
\mathscr{G}_{2}(x) \\
\mathscr{G}_{3}(x)
\end{array}\right) & =\left(\begin{array}{lll}
1 & & \\
& x q & \\
& & x q^{2}
\end{array}\right) \cdot\left(\begin{array}{l}
F_{1}^{\star}(x) \\
F_{2}^{\star}(x) \\
F_{3}^{\star}(x)
\end{array}\right) \\
& =\left(\begin{array}{lll}
1 & & \\
& x q & \\
& & x q^{2}
\end{array}\right) \cdot\left(\begin{array}{l}
F_{1}\left(x q^{2}\right) \\
F_{2}\left(x q^{2}\right) \\
F_{3}\left(x q^{2}\right)
\end{array}\right) .
\end{aligned}
$$

Also, (29) and (30) can be deduced with no difficulty.

\subsubsection{Example 2}

In the second example, we consider

"partitions with difference at least 3 at distance 2 such that if two consecutive parts differ by at most 1 , then their sum is divisible by $3 . "$

This partition set corresponds to the Kanade-Russell conjectures $I_{1}-I_{3}$. It was shown in [9] that this partition set is a span one linked partition ideal $\mathscr{I}(\langle\Pi, \mathcal{L}\rangle, S)$ where $S=3$, and $\Pi=\left\{\pi_{1}, \pi_{2}, \ldots, \pi_{7}\right\}$ along with the linking sets given as follows.

$$
\begin{array}{cc}
\Pi & \text { linking set } \\
\pi_{1}=\varnothing & \left\{\pi_{1}, \pi_{2}, \pi_{3}, \pi_{4}, \pi_{5}, \pi_{6}, \pi_{7}\right\} \\
\pi_{2}=1 & \left\{\pi_{1}, \pi_{2}, \pi_{3}, \pi_{4}, \pi_{5}, \pi_{6}, \pi_{7}\right\} \\
\pi_{3}=2+1 & \left\{\pi_{1}, \pi_{2}, \pi_{3}, \pi_{4}, \pi_{5}, \pi_{6}, \pi_{7}\right\} \\
\pi_{4}=3+1 & \left\{\pi_{1}, \pi_{5}, \pi_{6}, \pi_{7}\right\} \\
\pi_{5}=2 & \left\{\pi_{1}, \pi_{2}, \pi_{3}, \pi_{4}, \pi_{5}, \pi_{6}, \pi_{7}\right\} \\
\pi_{6}=3 & \left\{\pi_{1}, \pi_{5}, \pi_{6}, \pi_{7}\right\} \\
\pi_{7}=3+3 & \left\{\pi_{1}, \pi_{6}, \pi_{7}\right\}
\end{array}
$$


It was also shown in [9] that the generating function for such partitions is

$$
\left\{\begin{array}{c}
\mathscr{G}_{1}(x)+\mathscr{G}_{2}(x)+\mathscr{G}_{3}(x)+\mathscr{G}_{4}(x) \\
+\mathscr{G}_{5}(x)+\mathscr{G}_{6}(x)+\mathscr{G}_{7}(x)
\end{array}\right\}=\sum_{n_{1}, n_{2} \geqslant 0} \frac{q^{n_{1}^{2}+3 n_{2}^{2}+3 n_{1} n_{2}} x^{n_{1}+2 n_{2}}}{(q ; q)_{n_{1}}\left(q^{3} ; q^{3}\right)_{n_{2}}},
$$

that the generating function for such partitions with the smallest part at least 2 is

$$
\mathscr{G}_{1}(x)+\mathscr{G}_{5}(x)+\mathscr{G}_{6}(x)+\mathscr{G}_{7}(x)=\sum_{n_{1}, n_{2} \geqslant 0} \frac{q^{n_{1}^{2}+3 n_{2}^{2}+3 n_{1} n_{2}+n_{1}+3 n_{2}} x^{n_{1}+2 n_{2}}}{(q ; q)_{n_{1}}\left(q^{3} ; q^{3}\right)_{n_{2}}},
$$

and that the generating function for such partitions with the smallest part at least 3 is

$$
\mathscr{G}_{1}(x)+\mathscr{G}_{6}(x)+\mathscr{G}_{7}(x)=\sum_{n_{1}, n_{2} \geqslant 0} \frac{q^{n_{1}^{2}+3 n_{2}^{2}+3 n_{1} n_{2}+2 n_{1}+3 n_{2}} x^{n_{1}+2 n_{2}}}{(q ; q)_{n_{1}}\left(q^{3} ; q^{3}\right)_{n_{2}}} .
$$

We know from (26) that

$$
\left(\begin{array}{l}
F_{1}(x) \\
F_{2}(x) \\
F_{3}(x) \\
F_{4}(x) \\
F_{5}(x) \\
F_{6}(x) \\
F_{7}(x)
\end{array}\right)=\left(\begin{array}{lllllll}
1 & 1 & 1 & 1 & 1 & 1 & 1 \\
1 & 1 & 1 & 1 & 1 & 1 & 1 \\
1 & 1 & 1 & 1 & 1 & 1 & 1 \\
1 & 0 & 0 & 0 & 1 & 1 & 1 \\
1 & 1 & 1 & 1 & 1 & 1 & 1 \\
1 & 0 & 0 & 0 & 1 & 1 & 1 \\
1 & 0 & 0 & 0 & 0 & 1 & 1
\end{array}\right) \cdot\left(\begin{array}{l}
\mathscr{G}_{1}(x) \\
\mathscr{G}_{2}(x) \\
\mathscr{G}_{3}(x) \\
\mathscr{G}_{4}(x) \\
\mathscr{G}_{5}(x) \\
\mathscr{G}_{6}(x) \\
\mathscr{G}_{7}(x)
\end{array}\right) .
$$

Hence, by (34), (35) and (36), if we put

$$
\begin{gathered}
F_{1}(x)=F_{2}(x)=F_{3}(x)=F_{5}(x)=\sum_{n_{1}, n_{2} \geqslant 0} \frac{q^{n_{1}^{2}+3 n_{2}^{2}+3 n_{1} n_{2}} x^{n_{1}+2 n_{2}}}{(q ; q)_{n_{1}}\left(q^{3} ; q^{3}\right)_{n_{2}}}, \\
F_{4}(x)=F_{6}(x)=\sum_{n_{1}, n_{2} \geqslant 0} \frac{q^{n_{1}^{2}+3 n_{2}^{2}+3 n_{1} n_{2}+n_{1}+3 n_{2}} x^{n_{1}+2 n_{2}}}{(q ; q)_{n_{1}}\left(q^{3} ; q^{3}\right)_{n_{2}}}
\end{gathered}
$$

and

$$
F_{7}(x)=\sum_{n_{1}, n_{2} \geqslant 0} \frac{q^{n_{1}^{2}+3 n_{2}^{2}+3 n_{1} n_{2}+2 n_{1}+3 n_{2}} x^{n_{1}+2 n_{2}}}{(q ; q)_{n_{1}}\left(q^{3} ; q^{3}\right)_{n_{2}}},
$$

then we have the following relation from (25):

$$
\left(\begin{array}{l}
F_{1}(x) \\
F_{2}(x) \\
F_{3}(x) \\
F_{4}(x) \\
F_{5}(x) \\
F_{6}(x) \\
F_{7}(x)
\end{array}\right)=\left(\begin{array}{lllllll}
1 & 1 & 1 & 1 & 1 & 1 & 1 \\
1 & 1 & 1 & 1 & 1 & 1 & 1 \\
1 & 1 & 1 & 1 & 1 & 1 & 1 \\
1 & 0 & 0 & 0 & 1 & 1 & 1 \\
1 & 1 & 1 & 1 & 1 & 1 & 1 \\
1 & 0 & 0 & 0 & 1 & 1 & 1 \\
1 & 0 & 0 & 0 & 0 & 1 & 1
\end{array}\right) \cdot\left(\begin{array}{lllllll}
1 & & & & & & \\
& x q & & & & & \\
& & x^{2} q^{3} & & & & \\
& & & x^{2} q^{4} & & & \\
& & & & x q^{2} & & \\
& & & & & x q^{3} & \\
& & & & & & x^{2} q^{6}
\end{array}\right)\left(\begin{array}{l}
F_{1}\left(x q^{3}\right) \\
F_{2}\left(x q^{3}\right) \\
F_{3}\left(x q^{3}\right) \\
F_{4}\left(x q^{3}\right) \\
F_{5}\left(x q^{3}\right) \\
F_{6}\left(x q^{3}\right) \\
F_{7}\left(x q^{3}\right)
\end{array}\right) .
$$


Conversely, we are also able to recover

$$
\left(\mathscr{G}_{1}(x), \mathscr{G}_{2}(x), \mathscr{G}_{3}(x), \mathscr{G}_{4}(x), \mathscr{G}_{5}(x), \mathscr{G}_{6}(x), \mathscr{G}_{7}(x)\right)^{T}
$$

as well as (34), (35) and (36) provided that we have proved (40) directly since $F_{1}(0)=$ $F_{2}(0)=\cdots=F_{7}(0)=1$.

\subsection{A matrix factorization problem}

Motivated by (33) and (40), we turn our interest to a matrix factorization problem as follows.

Let $R$ be a positive integer. Let $\underline{\boldsymbol{\alpha}}=\left(\alpha_{i, j}\right) \in \operatorname{Mat}_{R \times R}(\mathbb{N})$ be a fixed symmetric matrix. Let $\underline{\mathbf{A}}=\left(A_{r}\right) \in \mathbb{N}_{>0}^{R}$ and $\underline{\gamma}=\left(\gamma_{r}\right) \in \mathbb{N}_{>0}^{R}$ be fixed.

Let $\mathfrak{F}$ be a set of $q$-multi-summations defined by

$$
\mathfrak{F}:=\left\{H(\underline{\boldsymbol{\beta}}): \underline{\boldsymbol{\beta}} \in \mathbb{Z}^{R} \text { and condition (43) is satisfied }\right\},
$$

where $H(\boldsymbol{\beta})=H\left(\beta_{1}, \ldots, \beta_{R}\right)$ is of the form

$$
H(\underline{\boldsymbol{\beta}}):=\sum_{n_{1}, \ldots, n_{R} \geqslant 0} \frac{q^{\sum_{r=1}^{R} \alpha_{r, r} n_{r}\left(n_{r}-1\right) / 2} q^{\sum_{1 \leqslant i<j \leqslant R} \alpha_{i, j} n_{i} n_{j}} q^{\sum_{r=1}^{R} \beta_{r} n_{r}} x^{\sum_{r=1}^{R} \gamma_{r} n_{r}}}{\left(q^{A_{1}} ; q^{A_{1}}\right)_{n_{1}} \cdots\left(q^{A_{R}} ; q^{A_{R}}\right)_{n_{R}}}
$$

and the additional condition reads: for all $\left(n_{1}, \ldots, n_{R}\right) \in \mathbb{N}^{R} \backslash\{(0,0, \ldots, 0)\}$,

$$
\sum_{r=1}^{R} \frac{\alpha_{r, r} n_{r}\left(n_{r}-1\right)}{2}+\sum_{1 \leqslant i<j \leqslant R} \alpha_{i, j} n_{i} n_{j}+\sum_{r=1}^{R} \beta_{r} n_{r}>0 .
$$

Now we consider a column functional vector

$$
\underline{\mathbf{F}}_{\underline{\boldsymbol{\beta}}}(x)=\left(\begin{array}{c}
F_{1}(x) \\
F_{2}(x) \\
\vdots \\
F_{K}(x)
\end{array}\right):=\left(\begin{array}{c}
H\left(\boldsymbol{\beta}_{1}\right) \\
H\left(\underline{\boldsymbol{\beta}}_{2}\right) \\
\vdots \\
H\left(\underline{\boldsymbol{\beta}}_{K}\right)
\end{array}\right),
$$

where $H\left(\underline{\boldsymbol{\beta}}_{k}\right) \in \mathfrak{F}$ for all $1 \leqslant k \leqslant K$.

We expect $\underline{\mathbf{F}}_{\underline{\boldsymbol{\beta}}}(x)$ to satisfy the following factorization property.

Factorization Property. Let $\mathscr{U}$ be a $(0,1)$-matrix such that all entries in the first row and column are 1 . Let $\mathscr{V}$ be a diagonal matrix such that all (diagonal) entries are monic monomials in $x$ and $q$ with $\mathscr{V}_{1,1}=1$. We say that $\underline{\mathbf{F}}_{\boldsymbol{\beta}}(x)$ satisfies the Factorization Property if

$$
\underline{\mathbf{F}}_{\boldsymbol{\beta}}(x)=\mathscr{U} \cdot \mathscr{V} \cdot \underline{\mathbf{F}}_{\boldsymbol{\beta}}\left(x q^{S}\right)
$$

for some positive integer $S$. 
Example 16. In the example in Section 3.2.1, we have $\underline{\boldsymbol{\alpha}}=(2), \underline{\boldsymbol{\gamma}}=(1), \underline{\mathbf{A}}=(1)$ and

$$
\underline{\mathbf{F}}_{\underline{\boldsymbol{\beta}}}(x)=\left(\begin{array}{l}
H(1) \\
H(1) \\
H(2)
\end{array}\right) .
$$

Also, $S=2$.

Example 17. In the example in Section 3.2 .2 , we have $\underline{\boldsymbol{\alpha}}=\left(\begin{array}{ll}2 & 3 \\ 3 & 6\end{array}\right), \underline{\boldsymbol{\gamma}}=(1,2), \underline{\mathbf{A}}=$ $(1,3)$ and

$$
\underline{\mathbf{F}}_{\underline{\boldsymbol{\beta}}}(x)=\left(\begin{array}{c}
H(1,3) \\
H(1,3) \\
H(1,3) \\
H(2,6) \\
H(1,3) \\
H(2,6) \\
H(3,6)
\end{array}\right) .
$$

Also, $S=3$.

\section{Non-computer-assisted proofs}

In [9], Li and the author provided an algebraic method to prove Andrews-Gordon type generating function identities such as (34), (35) and (36). However, we note that the proofs in that work rely heavily on computer assistance. Our aim here is to illuminate and simplify such proofs using an alternate approach.

As we have seen in Section 3.2.2, to prove (34), (35) and (36), it suffices to show (40). Our starting point is a recurrence relation enjoyed by $H\left(\beta_{1}, \ldots, \beta_{R}\right)$ defined in $(42)$.

\subsection{A key recurrence relation}

Recall that

$$
\begin{aligned}
& H\left(\beta_{1}, \ldots, \beta_{R}\right) \\
& \quad=\sum_{n_{1}, \ldots, n_{R} \geqslant 0} \frac{q^{\sum_{r=1}^{R} \alpha_{r, r} n_{r}\left(n_{r}-1\right) / 2} q^{\sum_{1 \leqslant i<j \leqslant R} \alpha_{i, j} n_{i} n_{j}} q^{\sum_{r=1}^{R} \beta_{r} n_{r}} x^{\sum_{r=1}^{R} \gamma_{r} n_{r}}}{\left(q^{A_{1}} ; q^{A_{1}}\right)_{n_{1}} \cdots\left(q^{A_{R}} ; q^{A_{R}}\right)_{n_{R}}} .
\end{aligned}
$$

Theorem 18. For $1 \leqslant r \leqslant R$, we have

$$
\begin{aligned}
H\left(\beta_{1}, \ldots, \beta_{r}, \ldots, \beta_{R}\right) & =H\left(\beta_{1}, \ldots, \beta_{r}+A_{r}, \ldots, \beta_{R}\right) \\
& +x^{\gamma_{r}} q^{\beta_{r}} H\left(\beta_{1}+\alpha_{r, 1}, \ldots, \beta_{r}+\alpha_{r, r}, \ldots, \beta_{R}+\alpha_{r, R}\right) .
\end{aligned}
$$


Proof. We have (recall that $\underline{\boldsymbol{\alpha}}$ is a symmetric matrix so that $\alpha_{i, j}=\alpha_{j, i}$ for $1 \leqslant i, j \leqslant R$ )

$$
\begin{aligned}
H & \left(\beta_{1}, \ldots, \beta_{r}, \ldots, \beta_{R}\right)-H\left(\beta_{1}, \ldots, \beta_{r}+A_{r}, \ldots, \beta_{R}\right) \\
& =\sum_{n_{1}, \ldots, n_{R} \geqslant 0} \frac{q^{\sum_{i} \alpha_{i, i} n_{i}\left(n_{i}-1\right) / 2} q^{\sum_{i<j} \alpha_{i, j} n_{i} n_{j}} q^{\sum_{i} \beta_{i} n_{i}}\left(1-q^{n_{r} A_{r}}\right) x^{\sum_{i} \gamma_{i} n_{i}}}{\left(q^{A_{1}} ; q^{A_{1}}\right)_{n_{1}} \cdots\left(q^{A_{r}} ; q^{A_{r}}\right)_{n_{r}} \cdots\left(q^{A_{R}} ; q^{A_{R}}\right)_{n_{R}}} \\
& =\sum_{\substack{n_{1}, \ldots, n_{R} \geqslant 0 \\
n_{r} \geqslant 1}} \frac{q^{\sum_{i} \alpha_{i, i} n_{i}\left(n_{i}-1\right) / 2} q^{\sum_{i<j} \alpha_{i, j} n_{i} n_{j}} q^{\sum_{i} \beta_{i} n_{i}} x^{\sum_{i} \gamma_{i} n_{i}}}{\left(q^{A_{1}} ; q^{A_{1}}\right)_{n_{1}} \cdots\left(q^{A_{r}} ; q^{A_{r}}\right)_{n_{r}-1} \cdots\left(q^{A_{R}} ; q^{A_{R}}\right)_{n_{R}}} \\
& =x^{\gamma_{r}} q^{\beta_{r}} \sum_{\substack{n_{1}, \ldots, n_{R} \geqslant 0\\
}} \frac{q^{\sum_{i} \alpha_{i, i} n_{i}\left(n_{i}-1\right) / 2} q^{\sum_{i<j} \alpha_{i, j} n_{i} n_{j}} q^{\sum_{i}\left(\beta_{i}+\alpha_{r, i}\right) n_{i}} x^{\sum_{i} \gamma_{i} n_{i}}}{\left(q^{A_{1}} ; q^{A_{1}}\right)_{n_{1}} \cdots\left(q^{A_{r}} ; q^{A_{r}}\right)_{n_{r}} \cdots\left(q^{A_{R}} ; q^{A_{R}}\right)_{n_{R}}} \\
& =x^{\gamma_{r}} q^{\beta_{r}} H\left(\beta_{1}+\alpha_{r, 1}, \ldots, \beta_{r}+\alpha_{r, r}, \ldots, \beta_{R}+\alpha_{r, R}\right) .
\end{aligned}
$$

The desired identity therefore follows.

Remark 19. It is worth pointing out that the recurrence (46) and its relations to sumlike generating functions have connections with the theory of vertex operator algebras, especially in the context of principal subspaces of modules. For one recent example, see (4.71)-(4.81) in [21].

Remark 20. A recent paper of Ablinger and Uncu [1] also seems to outline some functionality regarding recurrences for $q$-multi-summations.

Recall that the Factorization Property says that

$$
\underline{\mathbf{F}}_{\underline{\boldsymbol{\beta}}}(x)=\mathscr{U} \cdot \mathscr{V} \cdot \underline{\mathbf{F}}_{\underline{\boldsymbol{\beta}}}\left(x q^{S}\right) .
$$

Further, if $F(x)=H\left(\beta_{1}, \ldots, \beta_{R}\right)$, then

$$
F\left(x q^{S}\right)=H\left(\beta_{1}+\gamma_{1} S, \ldots, \beta_{R}+\gamma_{R} S\right) .
$$

Probably, if we expect to apply Theorem 18 to deduce Andrews-Gordon type generating function identities, we need to attach some additional conditions to the Factorization Property.

Additional Conditions. For all $1 \leqslant s \leqslant R$ :

(i). $\gamma_{s} S \in A_{s} \mathbb{Z}$;

(ii). for all $1 \leqslant r \leqslant R, \alpha_{r, s} \in A_{s} \mathbb{Z}$.

\subsection{Proof of (33)}

We first prove (33), which is relatively easy. 
Theorem 21. Let

$$
F_{1}(x)=F_{2}(x)=\sum_{n \geqslant 0} \frac{q^{n^{2}} x^{n}}{(q ; q)_{n}}
$$

and

$$
F_{3}(x)=\sum_{n \geqslant 0} \frac{q^{n^{2}+n} x^{n}}{(q ; q)_{n}}
$$

Then,

$$
\left(\begin{array}{l}
F_{1}(x) \\
F_{2}(x) \\
F_{3}(x)
\end{array}\right)=\left(\begin{array}{lll}
1 & 1 & 1 \\
1 & 1 & 1 \\
1 & 0 & 1
\end{array}\right) \cdot\left(\begin{array}{lll}
1 & & \\
& x q & \\
& & x q^{2}
\end{array}\right) \cdot\left(\begin{array}{l}
F_{1}\left(x q^{2}\right) \\
F_{2}\left(x q^{2}\right) \\
F_{3}\left(x q^{2}\right)
\end{array}\right)
$$

We have shown in Example 16 that in this case $S=2, \underline{\boldsymbol{\alpha}}=(2), \underline{\boldsymbol{\gamma}}=(1), \underline{\mathbf{A}}=(1)$ and

$$
\left(\begin{array}{l}
F_{1}(x) \\
F_{2}(x) \\
F_{3}(x)
\end{array}\right)=\left(\begin{array}{l}
H(1) \\
H(1) \\
H(2)
\end{array}\right)
$$

Further, it follows from (47) that

$$
F_{1}\left(x q^{2}\right)=F_{2}\left(x q^{2}\right)=H(3)
$$

and

$$
F_{3}\left(x q^{2}\right)=H(4)
$$

To prove (50), it suffices to show that

$$
F_{1}(x)=F_{1}\left(x q^{2}\right)+x q F_{2}\left(x q^{2}\right)+x q^{2} F_{3}\left(x q^{2}\right)
$$

and

$$
F_{3}(x)=F_{1}\left(x q^{2}\right)+x q^{2} F_{3}\left(x q^{2}\right) .
$$

It follows from Theorem 18 that

$$
\begin{aligned}
F_{1}(x) & =H(1) \\
& =\{H(1+1)+x q H(1+2)\} \\
& =H(2)+x q H(3) \\
& =\left\{H(2+1)+x q^{2} H(2+2)\right\}+x q H(3) \\
& =H(3)+x q^{2} H(4)+x q H(3)
\end{aligned}
$$




$$
=F_{1}\left(x q^{2}\right)+x q^{2} F_{3}\left(x q^{2}\right)+x q F_{2}\left(x q^{2}\right)
$$

Also,

$$
\begin{aligned}
F_{3}(x) & =H(2) \\
& =\left\{H(2+1)+x q^{2} H(2+2)\right\} \\
& =H(3)+x q^{2} H(4) \\
& =F_{1}\left(x q^{2}\right)+x q^{2} F_{3}\left(x q^{2}\right) .
\end{aligned}
$$

Identities (53) and (54) are therefore proved.

\subsection{Proof of (40)}

We next prove (40).

Theorem 22. Let

$$
\begin{gathered}
F_{1}(x)=F_{2}(x)=F_{3}(x)=F_{5}(x)=\sum_{n_{1}, n_{2} \geqslant 0} \frac{q^{n_{1}^{2}+3 n_{2}^{2}+3 n_{1} n_{2}} x^{n_{1}+2 n_{2}}}{(q ; q)_{n_{1}}\left(q^{3} ; q^{3}\right)_{n_{2}}}, \\
F_{4}(x)=F_{6}(x)=\sum_{n_{1}, n_{2} \geqslant 0} \frac{q^{n_{1}^{2}+3 n_{2}^{2}+3 n_{1} n_{2}+n_{1}+3 n_{2}} x^{n_{1}+2 n_{2}}}{(q ; q)_{n_{1}}\left(q^{3} ; q^{3}\right)_{n_{2}}}
\end{gathered}
$$

and

$$
F_{7}(x)=\sum_{n_{1}, n_{2} \geqslant 0} \frac{q^{n_{1}^{2}+3 n_{2}^{2}+3 n_{1} n_{2}+2 n_{1}+3 n_{2}} x^{n_{1}+2 n_{2}}}{(q ; q)_{n_{1}}\left(q^{3} ; q^{3}\right)_{n_{2}}} .
$$

Then,

$$
\left(\begin{array}{l}
F_{1}(x) \\
F_{2}(x) \\
F_{3}(x) \\
F_{4}(x) \\
F_{5}(x) \\
F_{6}(x) \\
F_{7}(x)
\end{array}\right)=\left(\begin{array}{lllllll}
1 & 1 & 1 & 1 & 1 & 1 & 1 \\
1 & 1 & 1 & 1 & 1 & 1 & 1 \\
1 & 1 & 1 & 1 & 1 & 1 & 1 \\
1 & 0 & 0 & 0 & 1 & 1 & 1 \\
1 & 1 & 1 & 1 & 1 & 1 & 1 \\
1 & 0 & 0 & 0 & 1 & 1 & 1 \\
1 & 0 & 0 & 0 & 0 & 1 & 1
\end{array}\right) \cdot\left(\begin{array}{lllllll}
1 & & & & & & \\
& x q & & & & & \\
& & x^{2} q^{3} & & & & \\
& & & x^{2} q^{4} & & & \\
& & & & x q^{2} & & \\
& & & & & x q^{3} & \\
& & & & & & x^{2} q^{6}
\end{array}\right)\left(\begin{array}{l}
F_{1}\left(x q^{3}\right) \\
F_{2}\left(x q^{3}\right) \\
F_{3}\left(x q^{3}\right) \\
F_{4}\left(x q^{3}\right) \\
F_{5}\left(x q^{3}\right) \\
F_{6}\left(x q^{3}\right) \\
F_{7}\left(x q^{3}\right)
\end{array}\right) .
$$

We have shown in Example 17 that in this case $S=3, \underline{\boldsymbol{\alpha}}=\left(\begin{array}{ll}2 & 3 \\ 3 & 6\end{array}\right), \underline{\boldsymbol{\gamma}}=(1,2)$, $\underline{\mathbf{A}}=(1,3)$ and

$$
\left(\begin{array}{l}
F_{1}(x) \\
F_{2}(x) \\
F_{3}(x) \\
F_{4}(x) \\
F_{5}(x) \\
F_{6}(x) \\
F_{7}(x)
\end{array}\right)=\left(\begin{array}{l}
H(1,3) \\
H(1,3) \\
H(1,3) \\
H(2,6) \\
H(1,3) \\
H(2,6) \\
H(3,6)
\end{array}\right) .
$$


Again, it follows from (47) that

$$
\begin{aligned}
F_{1}\left(x q^{3}\right)= & F_{2}\left(x q^{3}\right)=F_{3}\left(x q^{3}\right)=F_{5}\left(x q^{3}\right)=H(4,9), \\
F_{4}\left(x q^{3}\right) & =F_{6}\left(x q^{3}\right)=H(5,12)
\end{aligned}
$$

and

$$
F_{7}\left(x q^{3}\right)=H(6,12) .
$$

To prove (58), it suffices to show that

$$
\begin{gathered}
F_{1}(x)=\left\{\begin{array}{c}
F_{1}\left(x q^{3}\right)+x q F_{2}\left(x q^{3}\right)+x^{2} q^{3} F_{3}\left(x q^{3}\right)+x^{2} q^{4} F_{4}\left(x q^{3}\right) \\
+x q^{2} F_{5}\left(x q^{3}\right)+x q^{3} F_{6}\left(x q^{3}\right)+x^{2} q^{6} F_{7}\left(x q^{3}\right)
\end{array}\right\}, \\
F_{4}(x)=F_{1}\left(x q^{3}\right)+x q^{2} F_{5}\left(x q^{3}\right)+x q^{3} F_{6}\left(x q^{3}\right)+x^{2} q^{6} F_{7}\left(x q^{3}\right)
\end{gathered}
$$

and

$$
F_{7}(x)=F_{1}\left(x q^{2}\right)+x q^{3} F_{6}\left(x q^{3}\right)+x^{2} q^{6} F_{7}\left(x q^{3}\right) .
$$

We will adopt the following notation to make our argument more transparent. First, we underline a term to indicate that Theorem 18 is applied to this term. Also, if Theorem 18 is applied with respect to one coordinate, then that coordinate will be shown in boldface. Finally, two terms inside the braces are deduced by Theorem 18 from the overlined term in the previous line.

It follows from Theorem 18 that

$$
\begin{aligned}
F_{1}(x)= & \underline{H(1, \mathbf{3})} \\
= & \left\{\underline{H(\mathbf{1}, 6)}+x^{2} q^{3} H(4,9)\right\} \\
= & \{\underline{H(\mathbf{2}, 6)}+x q H(3,9)\}+x^{2} q^{3} H(4,9) \\
= & \left\{H(3,6)+x q^{2} H(4,9)\right\}+x q H(\mathbf{3}, 9)+x^{2} q^{3} H(4,9) \\
= & \underline{H(3, \mathbf{6})+x q^{2} H(4,9)+\left\{x q H(4,9)+x^{2} q^{4} H(5,12)\right\}+x^{2} q^{3} H(4,9)} \\
= & \left\{\underline{\{(\mathbf{3}, 9)}+x^{2} q^{6} H(6,12)\right\}+x q^{2} H(4,9)+x q H(4,9)+x^{2} q^{4} H(5,12) \\
& +x^{2} q^{3} H(4,9) \\
= & \left\{H(4,9)+x q^{3} H(5,12)\right\}+x^{2} q^{6} H(6,12)+x q^{2} H(4,9)+x q H(4,9) \\
& +x^{2} q^{4} H(5,12)+x^{2} q^{3} H(4,9) \\
= & F_{1}\left(x q^{3}\right)+x q^{3} F_{6}\left(x q^{3}\right)+x^{2} q^{6} F_{7}\left(x q^{3}\right)+x q^{2} F_{5}\left(x q^{3}\right)+x q F_{2}\left(x q^{3}\right) \\
& +x^{2} q^{4} F_{4}\left(x q^{3}\right)+x^{2} q^{3} F_{3}\left(x q^{3}\right) .
\end{aligned}
$$

Also,

$$
F_{4}(x)=\underline{H(2,6)}
$$




$$
\begin{aligned}
& =\left\{\underline{H(3, \mathbf{6})}+x q^{2} H(4,9)\right\} \\
& =\left\{\underline{H(\mathbf{3}, 9)}+x^{2} q^{6} H(6,12)\right\}+x q^{2} H(4,9) \\
& =\left\{H(4,9)+x q^{3} H(5,12)\right\}+x^{2} q^{6} H(6,12)+x q^{2} H(4,9) \\
& =F_{1}\left(x q^{3}\right)+x q^{3} F_{6}\left(x q^{3}\right)+x^{2} q^{6} F_{7}\left(x q^{3}\right)+x q^{2} F_{5}\left(x q^{3}\right) .
\end{aligned}
$$

Finally,

$$
\begin{aligned}
F_{7}(x) & =\underline{H(3, \mathbf{6})} \\
& =\left\{\underline{\{(\mathbf{3}, 9)}+x^{2} q^{6} H(6,12)\right\} \\
& =\left\{\underline{H(4,9)}+x q^{3} H(5,12)\right\}+x^{2} q^{6} H(6,12) \\
& =F_{1}\left(x q^{3}\right)+x q^{3} F_{6}\left(x q^{3}\right)+x^{2} q^{6} F_{7}\left(x q^{3}\right) .
\end{aligned}
$$

Identities (62), (63) and (64) are therefore proved.

\subsection{Binary trees}

Interestingly, the previous two proofs can be represented nicely by binary trees. More precisely, all nodes are of the form $H\left(\beta_{1}, \ldots, \beta_{r}, \ldots, \beta_{R}\right)$. Then Theorem 18 gives two children of $H\left(\beta_{1}, \ldots, \beta_{r}, \ldots, \beta_{R}\right)$ : the left child is $H\left(\beta_{1}, \ldots, \beta_{r}+A_{r}, \ldots, \beta_{R}\right)$, weighted by 1 , and the right child is $H\left(\beta_{1}+\alpha_{r, 1}, \ldots, \beta_{r}+\alpha_{r, r}, \ldots, \beta_{R}+\alpha_{r, R}\right)$, weighted by $x^{\gamma_{r}} q^{\beta_{r}}$. See Fig. 2; here, again, $\beta_{r}$ is shown in boldface since Theorem 18 is applied with respect to it.

Figure 2: Node $H\left(\beta_{1}, \ldots, \beta_{r}, \ldots, \beta_{R}\right)$ and its children

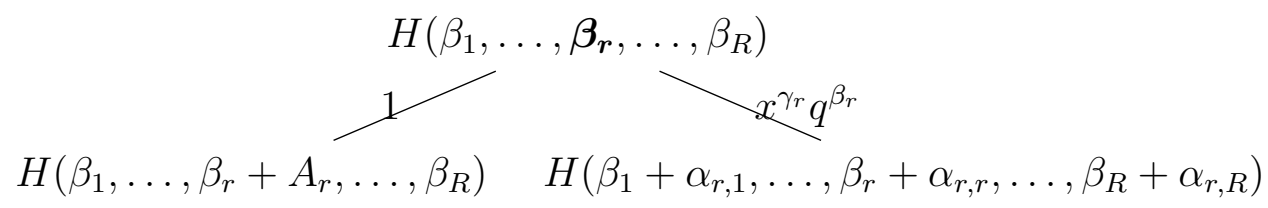

Now the proofs of (33) and (40) can be illustrated by Figs. 3 and 4, respectively.

Figure 3: The binary tree for (33)

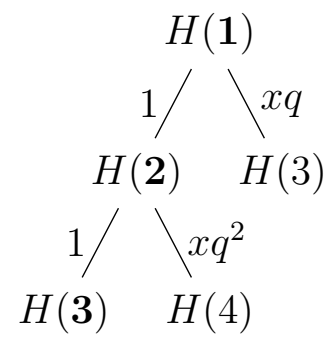

In fact, it is relatively easy to deduce other much more complicated identities of the same flavor as (33) and (40). For example, the next result, which appears to be new in literature, follows from the binary tree in Fig. 5. 
Figure 4: The binary tree for (40)

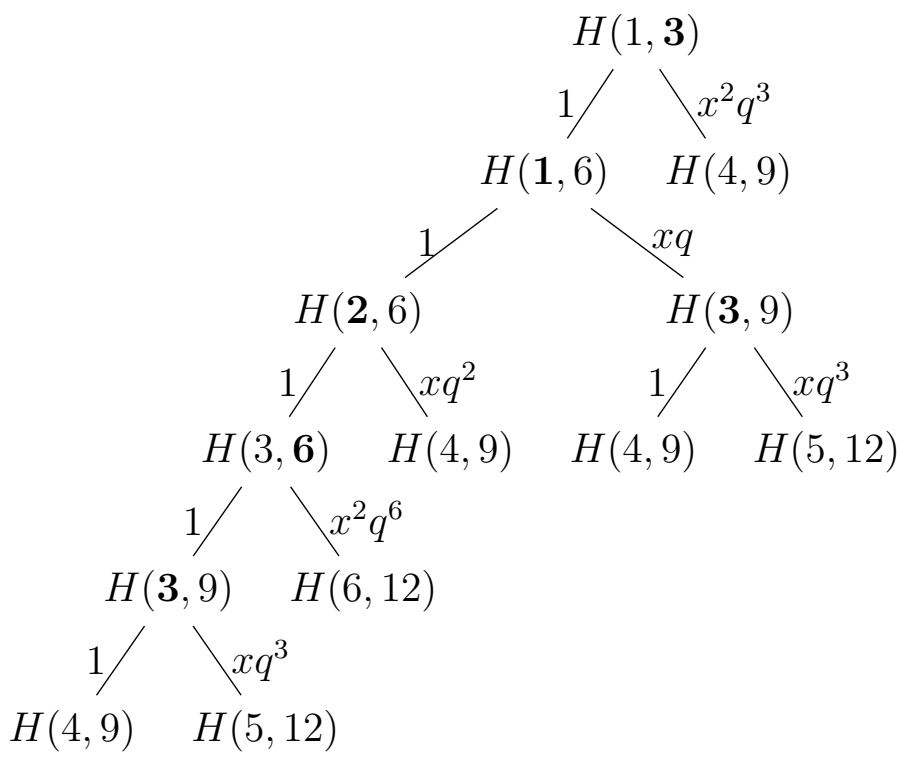

Theorem 23. Let

$$
\begin{aligned}
& F_{1}(x)=\cdots=F_{6}(x) \\
& =\sum_{n_{1}, n_{2}, n_{3} \geqslant 0} \frac{q^{\frac{n_{1}^{2}}{2}+3 n_{2}^{2}+\frac{9 n_{3}^{2}}{2}+2 n_{1} n_{2}+6 n_{2} n_{3}+3 n_{3} n_{1}+\frac{n_{1}}{2}-n_{2}-\frac{n_{3}}{2}} x^{n_{1}+2 n_{2}+3 n_{3}}}{(q ; q)_{n_{1}}\left(q^{2} ; q^{2}\right)_{n_{2}}\left(q^{3} ; q^{3}\right)_{n_{3}}}, \\
& F_{7}(x)=\cdots=F_{13}(x) \\
& =\sum_{n_{1}, n_{2}, n_{3} \geqslant 0} \frac{q^{\frac{n_{1}^{2}}{2}+3 n_{2}^{2}+\frac{9 n_{3}^{2}}{2}+2 n_{1} n_{2}+6 n_{2} n_{3}+3 n_{3} n_{1}+\frac{3 n_{1}}{2}+n_{2}+\frac{5 n_{3}}{2} x^{n_{1}+2 n_{2}+3 n_{3}}}}{(q ; q)_{n_{1}}\left(q^{2} ; q^{2}\right)_{n_{2}}\left(q^{3} ; q^{3}\right)_{n_{3}}}, \\
& F_{14}(x)=\cdots=F_{21}(x) \\
& =\sum_{n_{1}, n_{2}, n_{3} \geqslant 0} \frac{q^{\frac{n_{1}^{2}}{2}+3 n_{2}^{2}+\frac{9 n_{3}^{2}}{2}+2 n_{1} n_{2}+6 n_{2} n_{3}+3 n_{3} n_{1}+\frac{3 n_{1}}{2}+3 n_{2}+\frac{11 n_{3}}{2}} x^{n_{1}+2 n_{2}+3 n_{3}}}{(q ; q)_{n_{1}}\left(q^{2} ; q^{2}\right)_{n_{2}}\left(q^{3} ; q^{3}\right)_{n_{3}}}
\end{aligned}
$$

and

$$
\begin{aligned}
& F_{22}(x)=F_{23}(x) \\
& \quad=\sum_{n_{1}, n_{2}, n_{3} \geqslant 0} \frac{q^{\frac{n_{1}^{2}}{2}+3 n_{2}^{2}+\frac{9 n_{3}^{2}}{2}+2 n_{1} n_{2}+6 n_{2} n_{3}+3 n_{3} n_{1}+\frac{5 n_{1}}{2}+3 n_{2}+\frac{11 n_{3}}{2}} x^{n_{1}+2 n_{2}+3 n_{3}}}{(q ; q)_{n_{1}}\left(q^{2} ; q^{2}\right)_{n_{2}}\left(q^{3} ; q^{3}\right)_{n_{3}}} .
\end{aligned}
$$


Let

$$
\mathscr{A}=\left(\begin{array}{lllllllllllllllllllllll}
1 & 1 & 1 & 1 & 1 & 1 & 1 & 1 & 1 & 1 & 1 & 1 & 1 & 1 & 1 & 1 & 1 & 1 & 1 & 1 & 1 & 1 & 1 \\
1 & 1 & 1 & 1 & 1 & 1 & 1 & 1 & 1 & 1 & 1 & 1 & 1 & 1 & 1 & 1 & 1 & 1 & 1 & 1 & 1 & 1 & 1 \\
1 & 1 & 1 & 1 & 1 & 1 & 1 & 1 & 1 & 1 & 1 & 1 & 1 & 1 & 1 & 1 & 1 & 1 & 1 & 1 & 1 & 1 & 1 \\
1 & 1 & 1 & 1 & 1 & 1 & 1 & 1 & 1 & 1 & 1 & 1 & 1 & 1 & 1 & 1 & 1 & 1 & 1 & 1 & 1 & 1 & 1 \\
1 & 1 & 1 & 1 & 1 & 1 & 1 & 1 & 1 & 1 & 1 & 1 & 1 & 1 & 1 & 1 & 1 & 1 & 1 & 1 & 1 & 1 & 1 \\
1 & 1 & 1 & 1 & 1 & 1 & 1 & 1 & 1 & 1 & 1 & 1 & 1 & 1 & 1 & 1 & 1 & 1 & 1 & 1 & 1 & 1 & 1 \\
1 & 1 & 0 & 0 & 0 & 0 & 1 & 1 & 1 & 1 & 0 & 0 & 0 & 1 & 1 & 1 & 1 & 0 & 0 & 0 & 0 & 1 & 0 \\
1 & 1 & 0 & 0 & 0 & 0 & 1 & 1 & 1 & 1 & 0 & 0 & 0 & 1 & 1 & 1 & 1 & 0 & 0 & 0 & 0 & 1 & 0 \\
1 & 1 & 0 & 0 & 0 & 0 & 1 & 1 & 1 & 1 & 0 & 0 & 0 & 1 & 1 & 1 & 1 & 0 & 0 & 0 & 0 & 1 & 0 \\
1 & 1 & 0 & 0 & 0 & 0 & 1 & 1 & 1 & 1 & 0 & 0 & 0 & 1 & 1 & 1 & 1 & 0 & 0 & 0 & 0 & 1 & 0 \\
1 & 1 & 0 & 0 & 0 & 0 & 1 & 1 & 1 & 1 & 0 & 0 & 0 & 1 & 1 & 1 & 1 & 0 & 0 & 0 & 0 & 1 & 0 \\
1 & 1 & 0 & 0 & 0 & 0 & 1 & 1 & 1 & 1 & 0 & 0 & 0 & 1 & 1 & 1 & 1 & 0 & 0 & 0 & 0 & 1 & 0 \\
1 & 1 & 0 & 0 & 0 & 0 & 1 & 1 & 1 & 1 & 0 & 0 & 0 & 1 & 1 & 1 & 1 & 0 & 0 & 0 & 0 & 1 & 0 \\
1 & 1 & 0 & 0 & 0 & 0 & 1 & 1 & 0 & 0 & 0 & 0 & 0 & 1 & 1 & 1 & 0 & 0 & 0 & 0 & 0 & 1 & 0 \\
1 & 1 & 0 & 0 & 0 & 0 & 1 & 1 & 0 & 0 & 0 & 0 & 0 & 1 & 1 & 1 & 0 & 0 & 0 & 0 & 0 & 1 & 0 \\
1 & 1 & 0 & 0 & 0 & 0 & 1 & 1 & 0 & 0 & 0 & 0 & 0 & 1 & 1 & 1 & 0 & 0 & 0 & 0 & 0 & 1 & 0 \\
1 & 1 & 0 & 0 & 0 & 0 & 1 & 1 & 0 & 0 & 0 & 0 & 0 & 1 & 1 & 1 & 0 & 0 & 0 & 0 & 0 & 1 & 0 \\
1 & 1 & 0 & 0 & 0 & 0 & 1 & 1 & 0 & 0 & 0 & 0 & 0 & 1 & 1 & 1 & 0 & 0 & 0 & 0 & 0 & 1 & 0 \\
1 & 1 & 0 & 0 & 0 & 0 & 1 & 1 & 0 & 0 & 0 & 0 & 0 & 1 & 1 & 1 & 0 & 0 & 0 & 0 & 0 & 1 & 0 \\
1 & 1 & 0 & 0 & 0 & 0 & 1 & 1 & 0 & 0 & 0 & 0 & 0 & 1 & 1 & 1 & 0 & 0 & 0 & 0 & 0 & 1 & 0 \\
1 & 1 & 0 & 0 & 0 & 0 & 1 & 1 & 0 & 0 & 0 & 0 & 0 & 1 & 1 & 1 & 0 & 0 & 0 & 0 & 0 & 1 & 0 \\
1 & 0 & 0 & 0 & 0 & 0 & 1 & 0 & 0 & 0 & 0 & 0 & 0 & 1 & 1 & 0 & 0 & 0 & 0 & 0 & 0 & 1 & 0 \\
1 & 0 & 0 & 0 & 0 & 0 & 1 & 0 & 0 & 0 & 0 & 0 & 0 & 1 & 1 & 0 & 0 & 0 & 0 & 0 & 0 & 1 & 0
\end{array}\right)
$$

and

$$
\begin{aligned}
\mathscr{W}(x)=\operatorname{diag} & \left(1, x q^{2}, x q, x^{2} q^{3}, x^{2} q^{2}, x^{3} q^{4}\right. \\
& x q^{3}, x^{2} q^{5}, x^{2} q^{4}, x^{3} q^{7}, x^{2} q^{4}, x^{3} q^{6}, x^{3} q^{5} \\
& x^{2} q^{7}, x^{2} q^{6}, x^{3} q^{9}, x^{3} q^{8}, x^{3} q^{8}, x^{3} q^{7}, x^{4} q^{10}, x^{4} q^{9} \\
& \left.x^{3} q^{10}, x^{4} q^{11}\right) .
\end{aligned}
$$

Then,

$$
\left(\begin{array}{c}
F_{1}(x) \\
F_{2}(x) \\
\vdots \\
F_{23}(x)
\end{array}\right)=\mathscr{A} \cdot \mathscr{W}(x) \cdot\left(\begin{array}{c}
F_{1}\left(x q^{3}\right) \\
F_{2}\left(x q^{3}\right) \\
\vdots \\
F_{23}\left(x q^{3}\right)
\end{array}\right)
$$

Remark 24. It is worth pointing out that the $q$-multi-summations in this theorem are similar to those appear in $[17,(47)$ and (51)].

Proof of Theorem 23. Let $\underline{\boldsymbol{\alpha}}=\left(\begin{array}{lll}1 & 2 & 3 \\ 2 & 6 & 6 \\ 3 & 6 & 9\end{array}\right), \underline{\boldsymbol{\gamma}}=(1,2,3), \underline{\mathbf{A}}=(1,2,3)$ and $S=3$. We have

$$
F_{1}(x)=\cdots=F_{6}(x)=H(1,2,4) \quad \stackrel{x \mapsto x q^{3}}{\longrightarrow} \quad H(4,8,13)
$$




$$
\begin{array}{ccc}
F_{7}(x)=\cdots=F_{13}(x)=H(2,4,7) & \stackrel{x \mapsto x q^{3}}{\longrightarrow} \quad H(5,10,16), \\
F_{14}(x) & =\cdots=F_{21}(x)=H(2,6,10) \quad \stackrel{x \mapsto x q^{3}}{\longrightarrow} \quad H(5,12,19)
\end{array}
$$

and

$$
F_{22}(x)=F_{23}(x)=H(3,6,10) \quad \stackrel{x \mapsto x q^{3}}{\longrightarrow} \quad H(6,12,19) .
$$

The rest follows from the binary tree in Fig. 5.

It looks like one cannot deduce a span one linked partition ideal $\mathscr{I}(\langle\Pi, \mathcal{L}\rangle, S)$ from Theorem 23. This is because by (71), we need $S=3$. But in the diagonal matrix $\mathscr{W}(x)$, there is a term $x^{2} q^{7}$, which induces a partition of size 7 that has two parts. This means that one of the parts is larger than 3. However, for a span one linked partition ideal, we require that all parts in partitions among $\Pi$ must not exceed $S$.

On the other hand, we will show in the next corollary that Theorem 23 still corresponds to a partition set.

Corollary 25. Let $\Pi=\left\{\pi_{1}, \pi_{2}, \ldots, \pi_{23}\right\}$ be a set of integer partitions where

$$
\left[\begin{array}{llll}
\pi_{1}=\varnothing & \pi_{2}=2 & \pi_{3}=1 & \pi_{4}=2+1 \\
\pi_{5}=1+1 & \pi_{6}=2+1+1 & & \\
\hline \pi_{7}=3 & \pi_{8}=3+2 & \pi_{9}=2+2 & \pi_{10}=3+2+2 \\
\pi_{11}=3+1 & \pi_{12}=2+2+2 & \pi_{13}=3+1+1 & \\
\hline \pi_{14}=4+3 & \pi_{15}=3+3 & \pi_{16}=3+3+3 & \pi_{17}=3+3+2 \\
\pi_{18}=4+2+2 & \pi_{19}=3+2+2 & \pi_{20}=3+3+2+2 & \pi_{21}=3+2+2+2 \\
\hline \pi_{22}=4+3+3 & \pi_{23}=3+3+3+2 & &
\end{array}\right] .
$$

Let $\mathcal{L}: \Pi \rightarrow P(\Pi)$ where $P(\Pi)$ is the power set of $\Pi$ be defined by

$$
\mathcal{L}\left(\pi_{i}\right)= \begin{cases}\left\{\pi_{1}, \pi_{2}, \ldots, \pi_{23}\right\} & \text { for } 1 \leqslant i \leqslant 6 \\ \left\{\pi_{1}, \pi_{2}, \pi_{7}, \pi_{8}, \pi_{9}, \pi_{10}, \pi_{14}, \pi_{15}, \pi_{16}, \pi_{17}, \pi_{22}\right\} & \text { for } 7 \leqslant i \leqslant 13 \\ \left\{\pi_{1}, \pi_{2}, \pi_{7}, \pi_{8}, \pi_{14}, \pi_{15}, \pi_{16}, \pi_{22}\right\} & \text { for } 14 \leqslant i \leqslant 21 \\ \left\{\pi_{1}, \pi_{7}, \pi_{14}, \pi_{15}, \pi_{22}\right\} & \text { for } 22 \leqslant i \leqslant 23\end{cases}
$$

Let

$$
\mathcal{C}_{\lambda}: \lambda_{0} \rightarrow \lambda_{1} \rightarrow \lambda_{2} \rightarrow \cdots \rightarrow \lambda_{K} \rightarrow \varnothing \rightarrow \varnothing \rightarrow \cdots
$$

be a chain such that for all $i \geqslant 0, \lambda_{i} \in \Pi$ and $\lambda_{i+1} \in \mathcal{L}\left(\lambda_{i}\right)$. Let $\Phi_{\lambda}$ be an integer partition induced from $\mathcal{C}_{\lambda}$ defined as in (4) with $S=3$ :

$$
\Phi_{\lambda}=\lambda_{0} \oplus \phi^{3}\left(\lambda_{1}\right) \oplus \phi^{6}\left(\lambda_{2}\right) \oplus \cdots \oplus \phi^{3 K}\left(\lambda_{K}\right) \oplus \phi^{3(K+1)}(\varnothing) \oplus \phi^{3(K+2)}(\varnothing) \oplus \cdots .
$$

Let $\mathscr{S}$ be the set of such partitions $\Phi_{\lambda}$. Then,

$$
\sum_{v \in \mathscr{S}} x^{\sharp(v)} q^{|v|}=\sum_{n_{1}, n_{2}, n_{3} \geqslant 0} \frac{q^{\frac{n_{1}^{2}}{2}+3 n_{2}^{2}+\frac{9 n_{3}^{2}}{2}+2 n_{1} n_{2}+6 n_{2} n_{3}+3 n_{3} n_{1}+\frac{n_{1}}{2}-n_{2}-\frac{n_{3}}{2}} x^{n_{1}+2 n_{2}+3 n_{3}}}{(q ; q)_{n_{1}}\left(q^{2} ; q^{2}\right)_{n_{2}}\left(q^{3} ; q^{3}\right)_{n_{3}}} .
$$




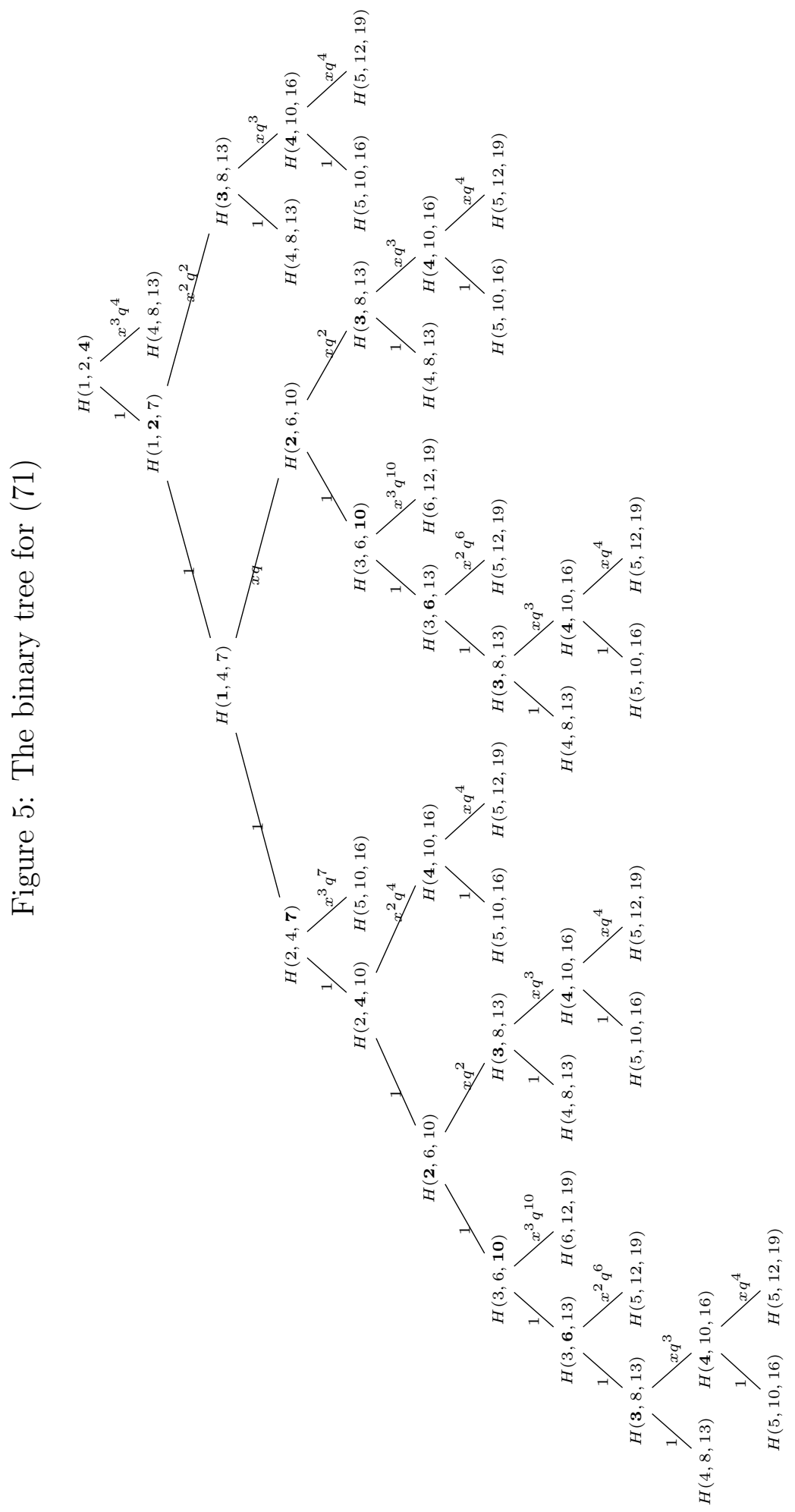

THE ELECTRONiC JOURNAL OF COMBINATORICS 27(3) (2020), \#3.33 
Proof. First, it is easy to see that given a chain $\mathcal{C}_{\lambda}$, the induced $\Phi_{\lambda}$ is indeed an integer partition. Now we claim that for any two chains $\mathcal{C}_{\mu}$ and $\mathcal{C}_{\nu}$, we have $\Phi_{\mu}=\Phi_{\nu}$ if and only if $\mathcal{C}_{\mu}=\mathcal{C}_{\nu}$. Notice that the "if" part is trivial.

We show the "only if" part by contradiction. Namely, we assume that there are two chains $\mathcal{C}_{\mu} \neq \mathcal{C}_{\nu}$ such that $\Phi_{\mu}=\Phi_{\nu}$. Let $\ell$ be the index such that $\mu_{\ell} \neq \nu_{\ell}$ and $\mu_{i}=\nu_{i}$ for $0 \leqslant i \leqslant \ell-1$. If neither $\mu_{\ell}$ nor $\nu_{\ell}$ contains a part of size 4 , then the parts in $\Phi_{\mu}$ of size up to $3(\ell+1)$ are given by $\oplus_{i=0}^{\ell} \phi^{3 i}\left(\mu_{i}\right)$ and similarly the parts in $\Phi_{\nu}$ of size up to $3(\ell+1)$ are given by $\oplus_{i=0}^{\ell} \phi^{3 i}\left(\nu_{i}\right)$. Since $\Phi_{\mu}=\Phi_{\nu}$ and $\mu_{i}=\nu_{i}$ for $0 \leqslant i \leqslant \ell-1$ as assumed, it follows that $\phi^{3 \ell}\left(\mu_{\ell}\right)=\phi^{3 \ell}\left(\nu_{\ell}\right)$ so that $\mu_{\ell}=\nu_{\ell}$. This contradicts the assumption that $\mu_{\ell} \neq \nu_{\ell}$. If 4 is a part in one of $\mu_{\ell}$ and $\nu_{\ell}$, then without loss of generality, we assume that 4 is a part in $\mu_{\ell}$. Then $\mu_{\ell} \in\left\{\pi_{14}, \pi_{18}, \pi_{22}\right\}$. Apparently, if 4 is also a part in $\nu_{\ell}$, we must have $\nu_{\ell}=\mu_{\ell}$, which violates the assumption. Now let us assume that 4 is not a part in $\nu_{\ell}$. Since $\Phi_{\mu}=\Phi_{\nu}$, we know that 1 must be a part in $\nu_{\ell+1}$; otherwise, $\Phi_{\nu}$ contains no parts of size $3 \ell+4$. Thus, $\nu_{\ell+1} \in\left\{\pi_{3}, \pi_{4}, \pi_{5}, \pi_{6}, \pi_{11}, \pi_{13}\right\}$. Since $\nu_{\ell+1} \in \mathcal{L}\left(\nu_{\ell}\right)$, we find that $\nu_{\ell} \in\left\{\pi_{1}, \pi_{2}, \pi_{3}, \pi_{4}, \pi_{5}, \pi_{6}\right\}$ and also the parts in $\Phi_{\nu}$ of size up to $3(\ell+1)$ are given by $\left(\oplus_{i=0}^{\ell-1} \phi^{3 i}\left(\nu_{i}\right)\right) \oplus \phi^{3 \ell}\left(\nu_{\ell}\right)$. On the other hand, since $\mu_{\ell} \in\left\{\pi_{14}, \pi_{18}, \pi_{22}\right\}$, the parts in $\Phi_{\mu}$ of size up to $3(\ell+1)$ are $\oplus_{i=0}^{\ell-1} \phi^{3 i}\left(\mu_{i}\right)$ plus one of $\phi^{3 \ell}(3), \phi^{3 \ell}(2+2)$ or $\phi^{3 \ell}(3+3)$ none of which could be $\phi^{3 \ell}\left(\nu_{\ell}\right)$. This implies that $\Phi_{\mu} \neq \Phi_{\nu}$, which leads to a contradiction.

Once we have shown that the induced partitions $\Phi_{\lambda}$ are pairwise distinct, the rest is a simple application of the framework developed in this paper by first constructing the associated directed graph as in Section 2.3. We leave this as an exercise to the interested reader.

\section{$5 \quad$ Closing remarks}

Our main concern is about the Factorization Property. Recall that $\mathscr{U}$ is a $(0,1)$-matrix such that all entries in the first row and column are 1 , and $\mathscr{V}$ is a diagonal matrix such that all (diagonal) entries are monic monomials in $x$ and $q$ with $\mathscr{V}_{1,1}=1$. The Factorization Property says that

$$
\underline{\mathbf{F}}_{\underline{\boldsymbol{\beta}}}(x)=\mathscr{U} \cdot \mathscr{V} \cdot \underline{\mathbf{F}}_{\underline{\boldsymbol{\beta}}}\left(x q^{S}\right),
$$

where $S$ is a positive integer and

$$
\underline{\mathbf{F}}_{\underline{\boldsymbol{\beta}}}(x)=\left(\begin{array}{c}
F_{1}(x) \\
F_{2}(x) \\
\vdots \\
F_{K}(x)
\end{array}\right)=\left(\begin{array}{c}
H\left(\boldsymbol{\beta}_{1}\right) \\
H\left(\underline{\boldsymbol{\beta}}_{2}\right) \\
\vdots \\
H\left(\underline{\boldsymbol{\beta}}_{K}\right)
\end{array}\right),
$$

in which $H(\underline{\boldsymbol{\beta}})=H\left(\beta_{1}, \ldots, \beta_{R}\right)$ is of the form

$$
H(\underline{\boldsymbol{\beta}})=\sum_{n_{1}, \ldots, n_{R} \geqslant 0} \frac{q^{\sum_{r=1}^{R} \alpha_{r, r} n_{r}\left(n_{r}-1\right) / 2} q^{\sum_{1 \leqslant i<j \leqslant R} \alpha_{i, j} n_{i} n_{j}} q^{\sum_{r=1}^{R} \beta_{r} n_{r}} x^{\sum_{r=1}^{R} \gamma_{r} n_{r}}}{\left(q^{A_{1}} ; q^{A_{1}}\right)_{n_{1}} \cdots\left(q^{A_{R}} ; q^{A_{R}}\right)_{n_{R}}} .
$$

Probably we also require the Additional Conditions: for all $1 \leqslant s \leqslant R$ : 
(i). $\gamma_{s} S \in A_{s} \mathbb{Z}$;

(ii). for all $1 \leqslant r \leqslant R, \alpha_{r, s} \in A_{s} \mathbb{Z}$.

Problem 26. For given $\mathscr{U}$ and $\mathscr{V}$, is it possible to determine if there exist $\underline{\mathbf{F}}_{\boldsymbol{\beta}}(x)$ and $S$ such that (73) is true?

We have another problem from a different direction.

Problem 27. Are there any criteria of $\underline{\mathbf{F}}_{\boldsymbol{\beta}}(x)$ that we are always able to find $\mathscr{U}, \mathscr{V}$ and $S$ such that (73) is true?

The last problem is probably simpler.

Problem 28. Can we construct a family of $\mathscr{U}, \mathscr{V}, \underline{\mathbf{F}}_{\boldsymbol{\beta}}(x)$ and $S$ such that (73) holds?

If we are able to find such a construction, then we may derive family of span one linked partition ideals (or at least a family of modified directed graphs) with nice analytic generation functions.

\section{Acknowledgements}

I am grateful to the referee for his/her careful reading and helpful comments.

\section{References}

[1] J. Ablinger and A. K. Uncu. qFunctions - A Mathematica package for $q$-series and partition theory applications. preprint (2019). arXiv:1910.12410.

[2] G. E. Andrews. An analytic proof of the Rogers-Ramanujan-Gordon identities. Amer. J. Math. 88 (1966), 844-846.

[3] G. E. Andrews. Partition identities. Advances in Math. 9 (1972), 10-51.

[4] G. E. Andrews. A general theory of identities of the Rogers-Ramanujan type. Bull. Amer. Math. Soc. 80 (1974), 1033-1052.

[5] G. E. Andrews. Problems and prospects for basic hypergeometric functions. Theory and application of special functions (Proc. Advanced Sem., Math. Res. Center, Univ. Wisconsin, Madison, Wis., 1975), pp. 191-224. Math. Res. Center, Univ. Wisconsin, Publ. No. 35, Academic Press, New York, 1975.

[6] G. E. Andrews. The Theory of Partitions. Reprint of the 1976 original. Cambridge Mathematical Library. Cambridge University Press, Cambridge, 1998. xvi+255 pp.

[7] K. Bringmann, C. Jennings-Shaffer, and K. Mahlburg. Proofs and reductions of various conjectured partition identities of Kanade and Russell. J. Reine Angew. Math., in press.

[8] S. Capparelli. On some representations of twisted affine Lie algebras and combinatorial identities. J. Algebra 154 (1993), no. 2, 335-355. 
[9] S. Chern and Z. Li. Linked partition ideals and Kanade-Russell conjectures. Discrete Math. 343 (2020), no. 7, 111876, 24 pp.

[10] B. Coulson, S. Kanade, J. Lepowsky, R. McRae, F. Qi, M. C. Russell, and C. Sadowski. A motivated proof of the Göllnitz-Gordon-Andrews identities. Ramanujan J. 42 (2017), no. 1, 97-129.

[11] H. Göllnitz. Partitionen mit differenzenbedingungen. J. Reine Angew. Math. 225 (1967), 154-190.

[12] B. Gordon. A combinatorial generalization of the Rogers-Ramanujan identities. Amer. J. Math. 83 (1961), 393-399.

[13] B. Gordon. Some continued fractions of the Rogers-Ramanujan type. Duke Math. J. 32 (1965), 741-748.

[14] J. Lepowsky and M. Zhu. A motivated proof of Gordon's identities. Ramanujan J. 29 (2012), no. 1-3, 199-211.

[15] S. Kanade, J. Lepowsky, M. C. Russell, and A. V. Sills. Ghost series and a motivated proof of the Andrews-Bressoud identities. J. Combin. Theory Ser. A 146 (2017), $33-62$.

[16] S. Kanade and M. C. Russell. IdentityFinder and some new identities of RogersRamanujan type. Exp. Math. 24 (2015), no. 4, 419-423.

[17] S. Kanade and M. C. Russell. Staircases to analytic sum-sides for many new integer partition identities of Rogers-Ramanujan type. Electron. J. Combin. 26 (2019), no. 1, Paper 1.6, 33 pp.

[18] M. Kauers and C. Koutschan. A Mathematica package for $q$-holonomic sequences and power series. Ramanujan J. 19 (2009), no. 2, 137-150.

[19] K. Kurşungöz. Andrews-Gordon type series for Kanade-Russell conjectures. Ann. Comb. 23 (2019), no. 3-4, 835-888.

[20] K. Kurşungöz. Andrews-Gordon type series for Capparelli's and Göllnitz-Gordon identities. J. Combin. Theory Ser. A 165 (2019), 117-138.

[21] M. Penn, C. Sadowski, and G. Webb. Principal subspaces of twisted modules for certain lattice vertex operator algebras. Internat. J. Math. 30 (2019), no. 10, 1950048, 47 pp.

[22] S. Ramanujan. Proof of certain identities in combinatory analysis. Proc. Cambridge Philos. Soc. 19 (1919), 214-216.

[23] A. Riese. qMultiSum - a package for proving $q$-hypergeometric multiple summation identities. J. Symbolic Comput. 35 (2003), no. 3, 349-376.

[24] L. J. Rogers. Third memoir on the expansion of certain infinite products. Proc. Lond. Math. Soc. 26 (1894), 15-32. 